UCRL-ID-134697

\title{
W1045 Environment on the Surfaces of the Drip Shield $\&$ Waste Package Outer Barrier
}

\author{
Gregory E. Gdowski
}

July 1999

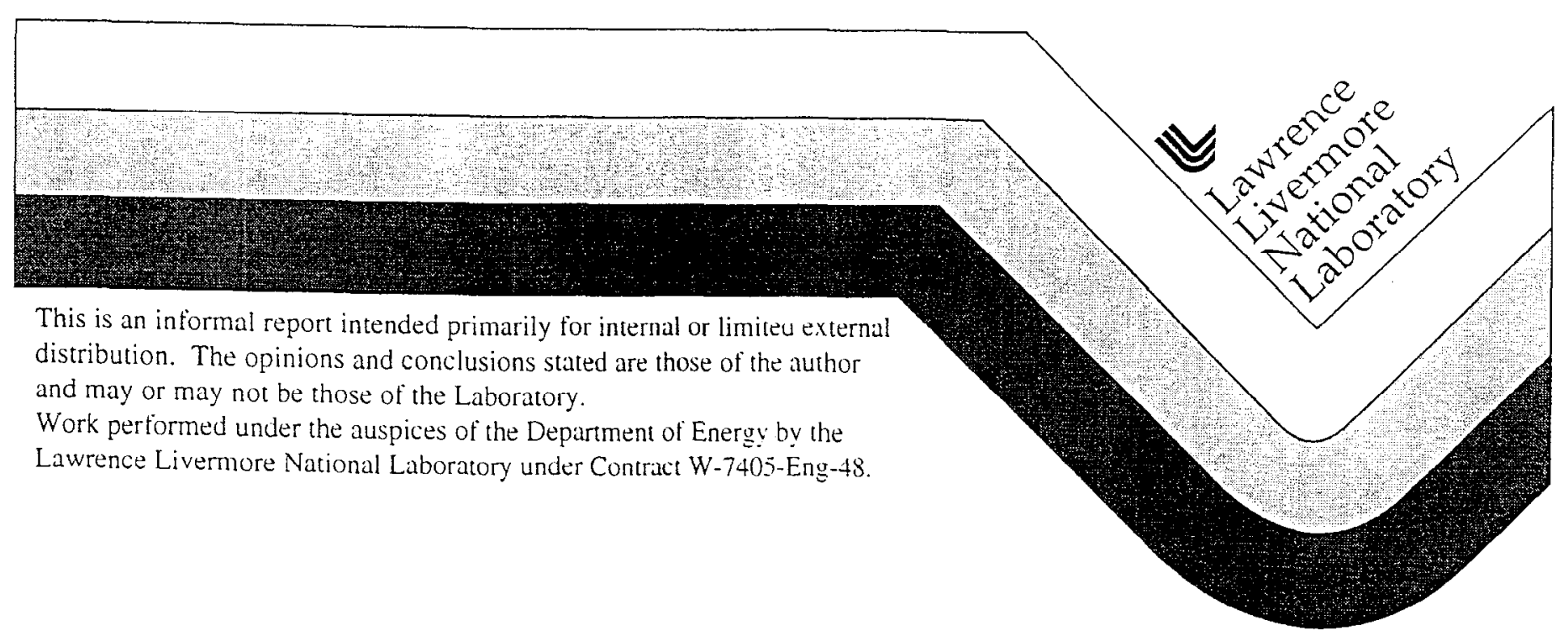


This document was prepared as an account of work sponsored by an agency of the United States Government. Neither the United States Govemment nor the University of Califormia nor any of their employees, makes any warranty, express or implied, or assumes any legal liability or responsibility for the accuracy, completeness, or usefulness of any information, apparatus, product, or process disclosed, or represents that its use would not infringe privately owned rights. Reference herein to any specific commercial product, process, or service by trade name, trademark, manufacturer, or otherwise, does not necessarily constitute or imply its endorsement, recommendation, or favoring by the United States Government or the University of Califormia. The views and opinions of authors expressed herein do not necessarily state or reflect those of the United States Govemment or the University of Califomia, and shall not be used for advertising or product endorsement purposes.

This report has been reproduced directly from the best available copy.

Available to DOE and DOE contractors from the Office of Scientific and Technical Information

P.O. Box 62, Oak Ridge, TN 37831

Prices available from (615) 576-8401, FTS 626-8401

Available to the public from the National Technical Information Service

U.S. Department of Commerce

5285 Port Royal Rd.

Springfield. VA 22161 


\title{
W1045 Environment on the Surfaces of the Drip Shield \& Waste Package Outer Barrier
}

\author{
ANL-EBS-MD-000001, Rev. 00A \\ by Gregory E. Gdowski
}

July 1999

Lawrence Livermore National Laboratory

Livermore, California 


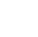




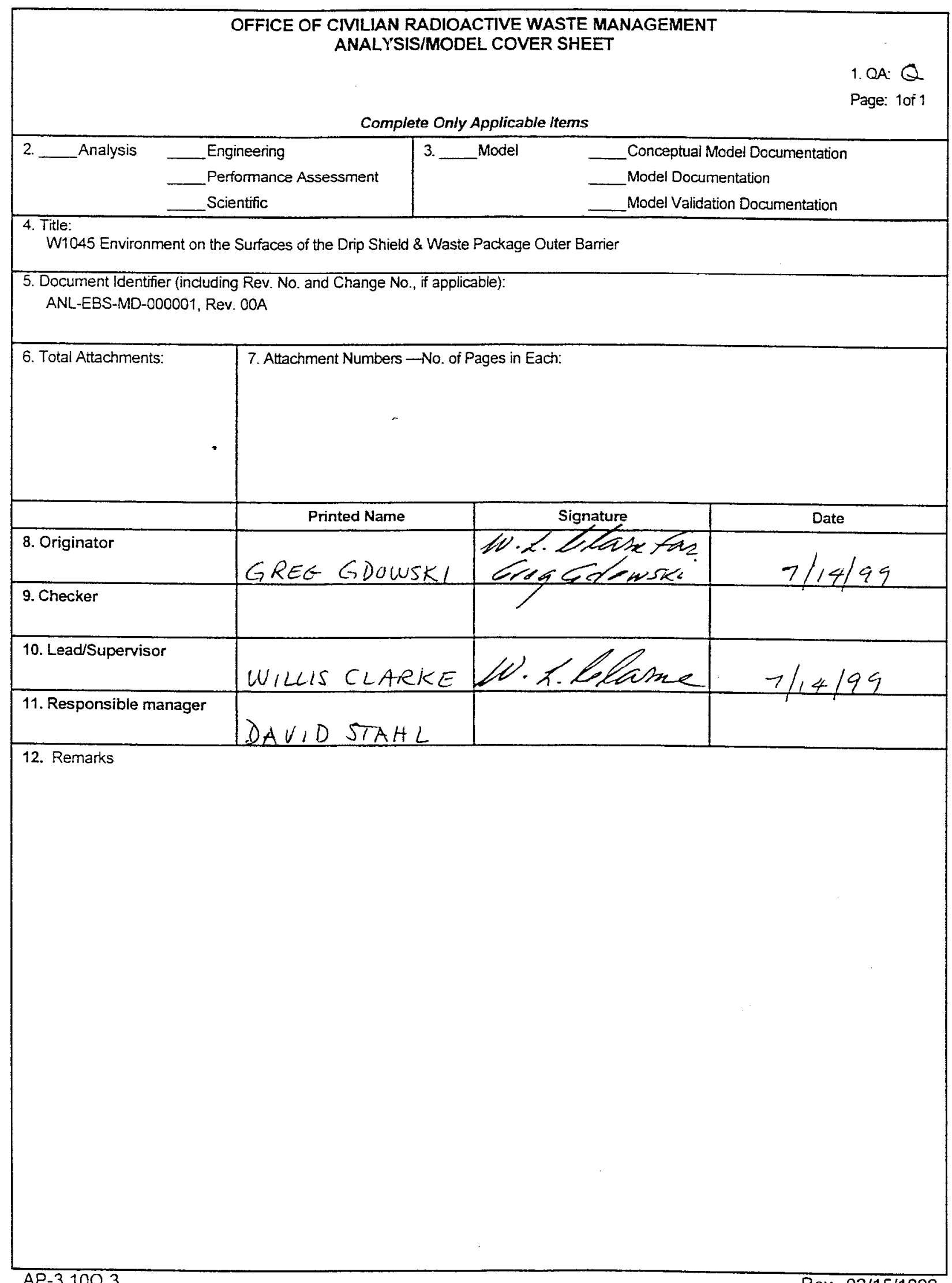




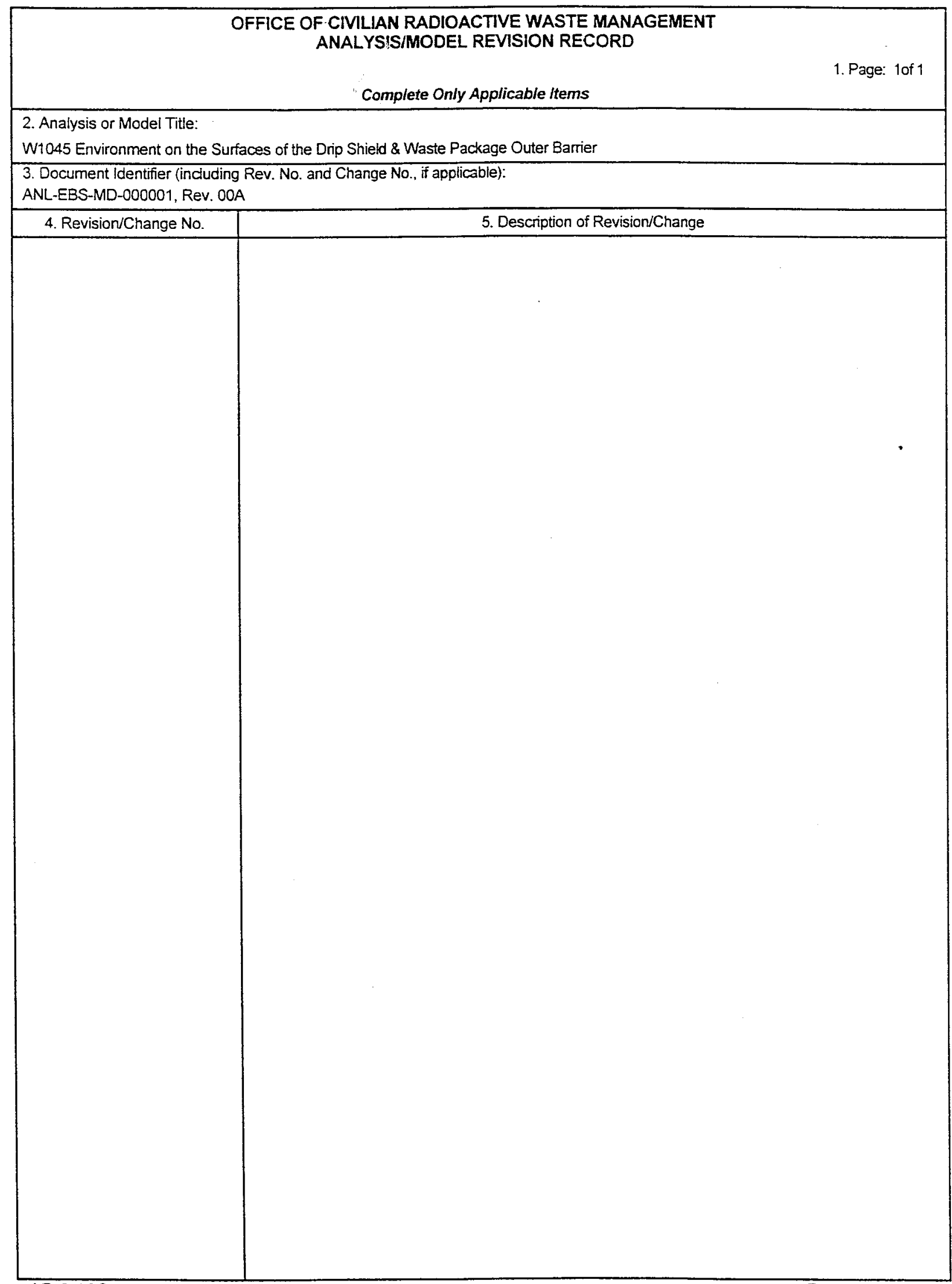




\section{CONTENTS}

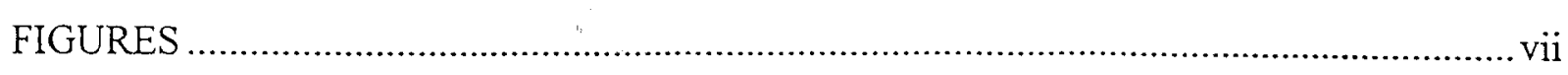

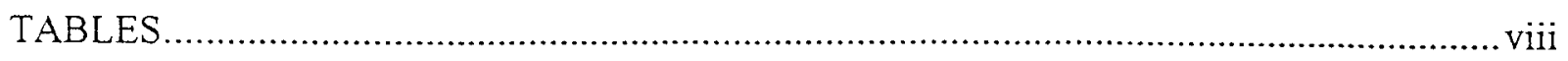

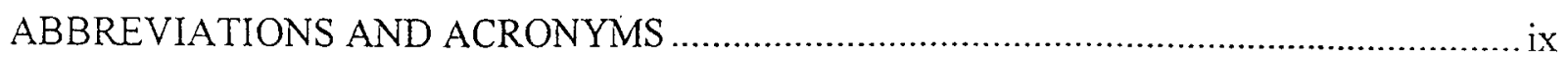

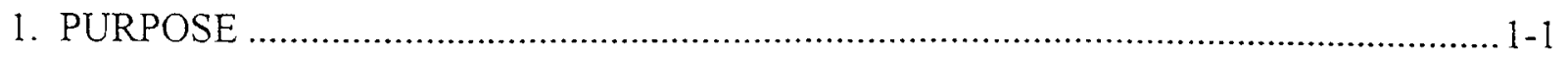

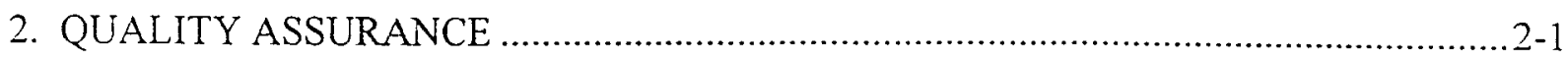

3. COMPUTER SOFTWARE AND MODEL USAGE ….................................................

3.1 Software Approved for QA Work ....................................................................... 3-1

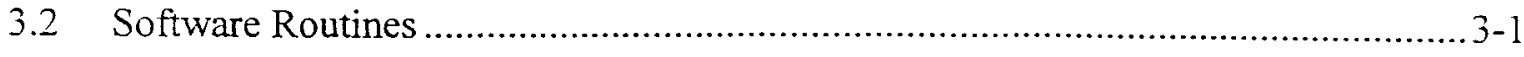

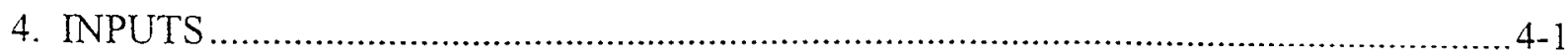

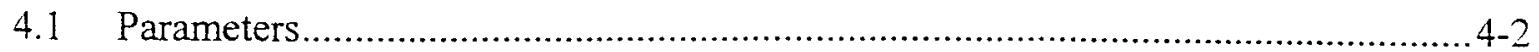

4.1.1 Components of Atmospheric Air Exclusive of Water .................................... 4-2

4.1.2 Water Vapor Pressure as a Function of Temperature .....................................4-2

4.1.3 Density of Water as a Function of Temperature ……...................................4-3

4.1.4 Surface Tension of Pure Water as a Function of Temperature .......................4-4

4.1.5 Atomic and Molecular Weights of Various Elements \& Molecules ..................4-4

4.1.6 Equilibrium Constant for Water Dissociation, $K_{W}$ …..................................4-5

4.1.7 Boiling Points of Saturated Solutions of Some Pure Salts................................4-6

4.1.8 Equilibrium Relative Humidity of Selected Saturated Salt Solutions

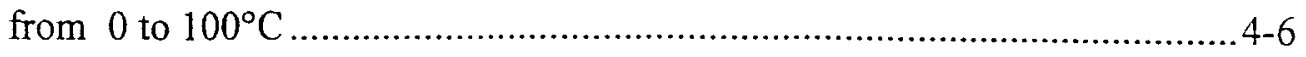

4.1.9 Solubilitics of $\mathrm{Ca}, \mathrm{Mg}, \mathrm{Na}$, and $\mathrm{K}$ Salts/Minerals in Water .............................4-6

4.1.10 Decomposition/Melting Temperatures of Some Bicarbonate/Carbonate

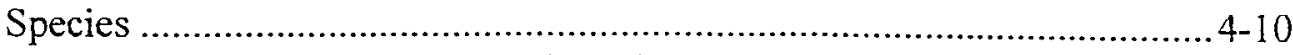

4.1.11 Adsorbed Water Films as a Function of Relative Humidity and

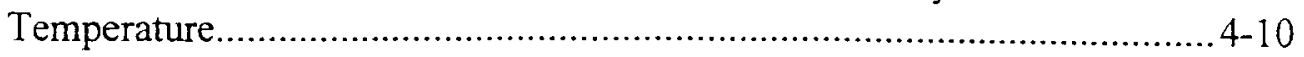

4.1.12 Vapor Pressure Lowering of Aqueous $\mathrm{NaCl}$ Solutions ................................ 4-11

4.1.13 Composition of Well J13 Water ............................................................. 4-13

4.1.14 Composition of Waters from Field Studies................................................ 4-14

4.1.15 Compositions of Perched and Pore Waters from Yucca Mountain................. 4-14

4.1.16 Compositions of Waters Contacting the Drip Shield and Waste Package Outer Barrier Surfaces ......................................................................... 4-14

4.1.17 Henry's Law Constants for the Solubility of Oxygen in Several Aqueous Solutions .......................................................................... 4-14

4.1.18 Evaporative/Boiling Concentrating of a Simulated Well J13 Water Near the Boiling Point of the Concentrated Water .............................................. 4-15

4.1.19 Evaporative/Boiling Concentrating of Waters from Field Studies Near the Boiling Point of the Concentrated Water ............................................... 4-16

4.1.20 Evaporative Concentrating of Simulated J13 at Temperatures Below the Boiling Point.......................................................................................... 4

4.1.21 Evaporative Concentrating of Waters from Field Studies at Temperatures Below the Boiling Point .....................................................4-17

4.1.22 Gas Phase Compositions in Drift...................................................... 4-17

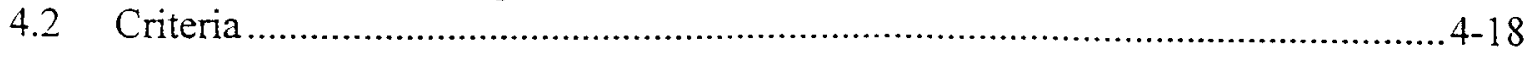


4.2.1 "System Environment Criteria".............................................................. 4-18

4.2.2 "System Performance Criteria" ................................................................. 4-18

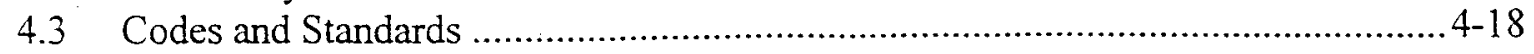

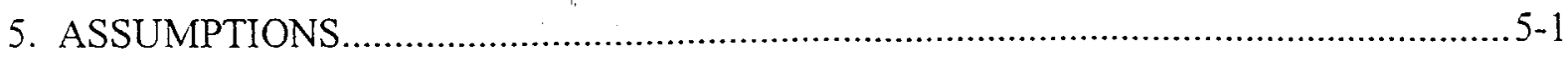

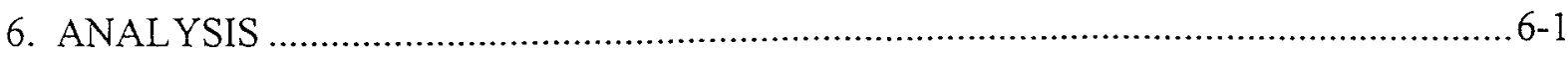

6.1 Introduction.................................................................................................

6.2 the $\mathrm{eH}$ and $\mathrm{pH}$ of Aqueous Solutions Under Relevant Repository Conditions ...........6-2

6.2.1 Accessible Relative Humidity Conditions ……….........................................6-2

6.2.2 Gas Phase Composition as a Function of Relative Humidity ….................... 6-2

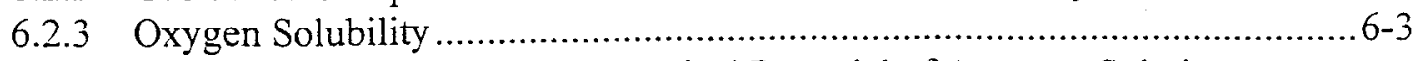

6.2.4 Determination of the Electrochemical Potential of Aqueous Solutions as

a Function of Oxygen Partial Pressure or Equivalently Oxygen

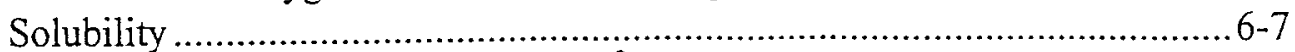

6.2.5 pH Control by the $\mathrm{CO}_{2}-\mathrm{HCO}_{3}-\mathrm{CO}_{3}{ }^{2-}$ System ....................................... 6-7

6.2.6 Quantify the Dependence of the Aqueous Solution $\mathrm{pH}$ on the Partial

Pressure of Carbon Dioxide as a Function of Temperature and Water

Partial Pressure ........................................................................................... 6-8

6.3 Water Adsorption on Surfaces in the Absence of Hygroscopic Salts .........................6-8

6.3.1 Water Adsorption on Flat Surfaces as a Function of Temperature and Relative Humidity .................................................................................. 6-8

6.3.2 Capillary Condensation as a Function of Relative Humidity and Temperature............................................................................................6-8

6.3.3 Relative Humidity Effect on the Oxidation and Dissolution of the Candidate Materials .............................................................................6-10

6.4 Aqueous Salt Solutions on the DSs and WPOBs................................................6-10

6.4.1 Deliquescence Points and Boiling Points for Pure Salts...............................6-11

6.4.2 RH Effect on Aqueous Solution Composition ...........................................6-12

6.4.3 Regions of Concern for the Aqueous Corrosion of DSs and WPOBs...........6-13

6.5 Boiling/Evaporative Concentrating of Simulated Well J13 Water ............................ 6-13

6.5.1 Initial Concentrating of Well J13 Water .................................................... 6-14

6.5.2 Evaporative Concentrating of a Simulated Well J13 by Boiling .................. 6-14

6.5.3 Water Composition as a Function of Relative Humidity at Fixed

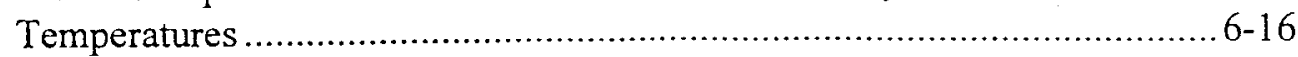

6.5.4 Salts/Minerals Formed Due to Evaporation of a Simulated J13 Water ......... 6-16

6.6 The Existence of $r$ queous Solutions on The EBS Components Under

Environment Conditions That Do Not Support Those Solutions ............................ 6-17

6.7 Estimate of the Composition of Corrosively Aggressive Aqueous Solution That Coutd Result from Concentrating Yucca Mountain Waters......................................6-17

6.8 Characterization of Mineral/Salt Scale Formation on Metal Surfaces...................... 6-17

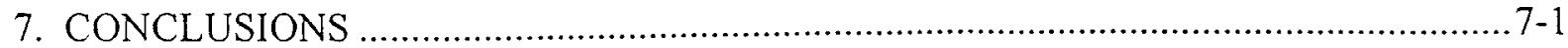

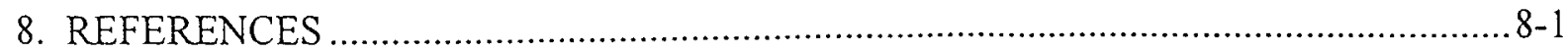

9. ATTACHMENTS................................................................................................ 


\section{CHECK COPY}

\section{FIGURES}

Figure 4-1. Amount of Water Adsorbed on a Nickel as a Function of Temperature (20 to $\left.80^{\circ} \mathrm{C}\right)$

Figure 4-2. Amount of Water Adsorbed on a Nickel as a Function of Relative Humidity (20 to $98 \% \mathrm{RH}$ )

Figure 6-1. Maximum Relative Humidity versus Temperature for Boiling Points of $96^{\circ} \mathrm{C}$ and $100^{\circ} \mathrm{C}$

Figure 6-2. Calculated Partial Pressure of $\mathrm{O}_{2}$ and $\mathrm{CO}_{2}$ Assuming $\mathrm{H}_{2} \mathrm{O}$ Partial Pressure is at its Maximum; That is, $100 \% \mathrm{RH}$ for $\mathrm{T} \leq 100^{\circ} \mathrm{C}$ and $101.32 \mathrm{kPa}$ for $\mathrm{T}>100^{\circ} \mathrm{C}$

Figure 6-3. Calculations of $\mathrm{O}_{2}$ and $\mathrm{CO}_{2}$ Partial Pressures Assuming $\mathrm{RH}=90 \%$ for

$\mathrm{T}<100^{\circ} \mathrm{C}$ and the Partial Pressure of Water is Equal to 0.9 Times the Total System Pressure Above $100^{\circ} \mathrm{C}$

Figure 6-4. Dissolved Oxygen Concentration (ppm) for Three Aqueous Solutions. Water

Partial Pressure: $100 \% \mathrm{RH}$ for $\mathrm{T} \leq 100^{\circ} \mathrm{C}$ and $101.32 \mathrm{kPa}$ for $\mathrm{i}>100^{\circ} \mathrm{C}$

Figure 6-5. Dissolved Oxygen Concentration (ppm) for Three Aqueous Solutions. Partial

Pressure of Water Determined by: $90 \% \mathrm{RHI}$ for $\mathrm{T} \leq 100^{\circ} \mathrm{C}$ and 87.67 for $\mathrm{T}>100^{\circ} \mathrm{C}$

Figure 6-6. Deliquescence Points and Boiling Points for Several Pure Salts 6-12

Figure 6-7. Aqueous $\mathrm{NaCl}$ Molality as a Function of Fractional Relative Humidity. $6-13$

Figure 6-8. Concentration Factors for the Cations for the Evaporative Concentration of Simulated Well J13 Water Near the Boiling Point. $6-15$

Figure 6-9. Concentration Factors for the Anions for the Evaporative Concentration of Simulated Well J13 Water Near the Boiling Point. 


\section{CHECK COPY}

\section{TABLES}

Page

Table 4-1. Components of Atmospheric Air Exclusive of Water Vapor..............................4-2

Table 4-2. Water Vapor Pressure as a Function of Temperature ........................................ 4-2

Table 4-3. Density of Pure Water as a Function of Temperature .......................................... 4-3

Table 4-4. Surface Tension of Pure Water as a Function of Temperature...............................4-4

Table 4-5. Listing of the Atomic and Molecular Weights of Elements and Molecules

Relevant to the Constituents in Yucca Mountain Waters....................................4-4

Table 4-6. Equilibrium Water Dissociation Constant as a Function of Temperature ...............4-5

Table 4-7. Boiling Points of Aqueous Solutions of Pure Salts ..........................................4-6

Table 4-8. Equilibrium Relative Humidity of Saturated Aqueous Solutions of Various

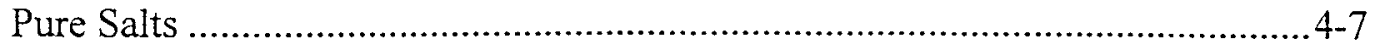

Table 4-9. Solubilities of $\mathrm{Ca}, \mathrm{Mg}, \mathrm{Na}$, and $\mathrm{K}$ Salts/Minerals in Water ..................................4-8

Table 4-10. Decomposition/Melting Temperatures of Relevant Bicarbonate/Carbonate Species ......................................................................................... 4-10

Table 4-11. Experimental Data on the Vapor Pressure Lowering of Aqueous $\mathrm{NaCl}$ Solutions as a Function of Temperature ..................................................... 4-12

Table 4-12. $\mathrm{NaCl}$ Molality as a Function of Temperature ............................................... 4-13

Table 4-13. Composition of Well J13 Water ..............................................................4-13

Table 4-14. Coefficients for Henry's Law Constants for the Oxygen Solubility in the $\mathrm{NaCl}$ Solutions and Demineralized Water....................................................4-14

Table 4-15. Calculated Henry's Law Constants for Oxygen Solubility in Three Aqueous

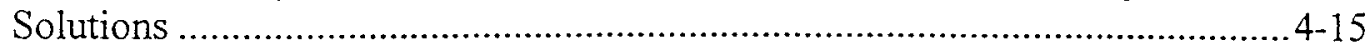

Table 4-16. The Compositions of Evaporatively Concentrated Simulated 100x Well J13 Water .................................................................................................. 4-16

Table 4-17. Results of the Evaporative Concentration of a Simulated Well J13 Water Under Conditions of Constant Temperature and Relative Humidity ................... 4-17

Table 4-18. Emplacement Drift External Environment..................................................... 4-18

Table 6-1. Calculation of the Radius of a Pore in which Water Condensation will Occur. ....... 6-9

Table 6-2. Results of the Evaporative Concentration of Simulated Well J13 Water Under Conditions of Constant Temperature and Relative Humidity ............................6-16

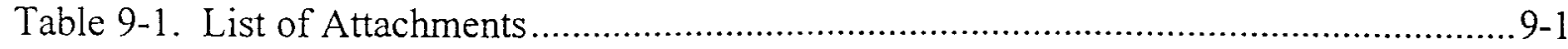




\section{ABBREVIATIONS AND ACRONYMS}

DS drip shield

$a_{0}, a_{1}, a_{3}, a_{+}$experimentally determined constants for calculation of Henry's Law constant

Eh electrochemical potential

EBS engineered barrier system

$D_{O 2}(p p m)$ solubility of oxygen in parts per million (ppm) by weight

$k \quad$ Henry's Law constant

MGR monitored geologic repository

$m$ molality (moles of solute $/ 1000 \mathrm{gm}$ of solvent)

$M W_{H 2 O} \quad$ molecular weight of water $(18.0153 \mathrm{gm} / \mathrm{gm}-\mathrm{mol})$

$M W_{O_{2}} \quad$ molecular weight of $\mathrm{O}_{2}$

$M_{\text {solute }} \quad$ molarity (moles of the solute/liter of solution)

$P_{\text {tmal }} \quad$ total pressure of the air

$P^{0} \quad$ vapor pressure of pure substance

$P \quad$ partial pressure

$p H \quad$ negative logarithm of the hydrogen ion concentration

ppm parts per million

RH r lative humidity $\left(P_{\mathrm{H} 2 \mathrm{O}} / P_{\mathrm{H} 2 \mathrm{O}}^{0}\right)$

QA quality assurance

$T \quad$ temperature $(\mathrm{K})$

$x_{\mathrm{CO} 2}$ air fraction of carbon dioxide

$x_{02} \quad$ air fraction of oxygen

$x_{R H} \quad$ fractional relative humidity 
$V P_{H 2 O} \quad$ water vapor pressure

$\rho_{s i \text { ln }} \quad$ density of the solution in gm/liter

WP waste package

WPOB waste package outer barrier 


\section{CHECK COPY}

\section{PURPOSE}

These analyses provide information on the chemistry of the water films that form on drip shield (DS) and waste package outer barrier (WPOB) surfaces as a function of temperature, relative humidity, and gas phase composition. Water vapor is not included in the gas phase composition but is considered separately as the relative humidity. The analyses also provide information on the types of mineral/salt scale that could form on DS and WPOB surfaces. These analyses assume that the composition of in-drift water is similar to that of well $\mathrm{J} 13$ water or concentrated well $\mathrm{J} 13$ water. Well $\mathrm{J} 13$ water is assumed to be representative of the ambient conditions at the proposed repository horizon at Yucca Mountain, Nevada. The analyses can be modiafed if a different water chemistry for the seepage into the drift is determined to be more relevant; that is, one that accounts for changes due to interactions with the near field after waste emplacement. 


\section{CHECK COPY}




\section{QUALITY ASSURANCE}

The quality assurance (QA) program applies to the development of this report. The data provided in this report will be used for evaluating the post-closure performance of the monitored geologic repository (MGR) waste package and engineered barrier segment. The QAP-2-3 (Classification of Permanent Items) evaluation entitled Classification of the Preliminary MGDS Repository Design (CRWMS M\&O 1998a, p. IV-11) has identified the waste package as a MGR (formerly MGDS) item important to safety and waste isolation. The waste package responsible manager has evaluated the technical document development activity in accordance with QAP-2-0, Conduct of Activities. The QAP-2-0 activity evaluation, Commercial SNF WP Reference Designs (CRWMS M\&O 1999a), has determined that the preparation and review of this technical document is subject to Quality Assurance Requirements and Description (DOE 1998a) requirements. In accordance with AP-3.10Q, a work plan was developed, issued, and used in the preparation of this document (CRWMS M\&O 1999b). 
$=$ 
3. COMPUTER SOFTWARE AND MODEL USAGE

\subsection{SOFTWARE APPROVED FOR QA WORK}

None used.

\subsection{SOFTWARE ROUTINES}

Microsoft Excel 98 (Microsoft Office 98), loaded on a PowerMac G-3 with Mac OS 8.5.1. Calculations were performed electronically in this spreadsheet software package. The equations for which the calculations were performed are included in the text of the report. Electronic media containing the files of the calculations will be submitted as part of the records package. 


\section{CHECK COPY}

\section{INPUTS}

The following 3.10Q AMRs provide necessary inputs for the analyses of this 3.10Q AMR.

Physical and Chemical Environment Model AP-3.10Q; EB35; RPPM100A

Responsible Organization: PAO

Due Date: 2 Dec 99

Seepage/Invert Interactions AP-3.10Q; EB215; RPPM035A

Responsible Organization: PAO

Due Date: 30 Aug 99

Seepage/Backfill Interactions AP-3.10Q; EB165; RPPM015

Responsible Organization: PAO

Due Date: 10 Aug 99

Precipitates/Salts Analysis AP-3.10Q; EB45; RPPM105A;

Responsible Organization: PAO

Due Date: 7 Oct 99

In-Drift Gas Flux \& Composition AP-3.10Q; EB175; RPPM055A;

Responsible Organization: PAO

Due Date: 4 Oct 99

Seepage/Cement AP-3.10Q; EB208; RPPM030A;

(has this been change to interaction $w /$ drift support materials A36 carbon steel)

Responsible Organization: PAO

Due Date: 18 Oct 99

Analysis of THC Processes/impacts on emplacement drift water chemistry/gas comp; N3010; SPP7106;

Responsible Organization: NEPO

Due Date: 31 Aug 99

Description of base case results of NFE thermodynamic environment including THC and THM; N3030; SL2000M4;

Responsible Organization: NEPO

Due Date: 02 Oct 99

Base case results of NFE for in-drift water chemistry/gas comp; N3040; SL20002M4;

Responsible Organization: NEPO

Due Date: 30 Sep 99 


\section{CHECK COPY}

The following $3.10 \mathrm{Q}$ report is not currently used in this $3.10 \mathrm{Q}$ report. However, if the information is relevant it will be incorporated in later versions.

Microbial Communities AP03.10Q; EB185; RPPM020A

Responsible Organization: PAO

Due Date: 14 Sep 99

\subsection{PARAMETERS}

\subsubsection{Components of Atmospheric Air Exclusive of Water}

The components of atmospheric air exclusive of water vapor is given in Table 4-1.

Table 4-1. Components of Atmospheric Air Exclusive of Water Vapor (Weast and Astle 1981)

\begin{tabular}{|c|c|c|}
\hline Constituent & Content (\%) by Volume & Content (ppm) by Volume \\
\hline $\mathrm{N}_{2}$ & $78.084 \pm 0.004$ & \\
\hline $\mathrm{O}_{2}$ & $20.946 \pm 0.002$ & \\
\hline $\mathrm{CO}_{2}$ & $0.033 \pm 0.001$ & \\
\hline $\mathrm{Ar}$ & $0.934 \pm 0.001$ & \\
\hline $\mathrm{Ne}$ & & $18.18 \pm 0.04$ \\
\hline $\mathrm{He}$ & & $5.24 \pm 0.004$ \\
\hline $\mathrm{Kr}$ & & $1.14 \pm 0.01$ \\
\hline $\mathrm{Xe}$ & & $0.087 \pm 0.001$ \\
\hline $\mathrm{H}_{2}$ & & 0.5 \\
\hline $\mathrm{CH}_{4}$ & & 2 \\
\hline $\mathrm{N}_{2} \mathrm{O}$ & & $0.5 \pm 0.1$ \\
\hline
\end{tabular}

\subsubsection{Water Vapor Pressure as a Function of Temperature}

The water vapor pressure as a function of temperature is listed in Table 4-2. The data were taken from Weast and Astle (1981). The vapor pressure was given in Torr. The vapor pressure is also given in $\mathrm{kPa}$ by applying the conversion factor 760 torr $=101.32 \mathrm{kPa}$.

Table 4-2. Water Vapor Pressure as a Function of Temperature (Weast and Astle 1981)

\begin{tabular}{|c|c|c|c|c|c|}
\hline Temp $^{\circ} \mathrm{C}$ & $\mathbf{k P}_{\mathbf{a}}$ & torr $^{*}$ & Temp $^{\circ} \mathrm{C}$ & $\mathbf{k P a}$ & torr $^{*}$ \\
\hline 0 & 0.61 & 4.59 & 155 & 543.38 & 4075.88 \\
\hline 5 & 0.87 & 6.55 & 160 & 618.05 & 4636.00 \\
\hline 10 & 1.23 & 9.21 & 165 & 700.73 & 5256.16 \\
\hline 15 & 1.71 & 12.79 & 170 & 792.02 & 5940.92 \\
\hline 20 & 2.34 & 17.54 & 175 & 892.43 & 6694.08 \\
\hline 25 & 3.17 & 23.77 & 180 & 1002.56 & 7520.20 \\
\hline 30 & 4.25 & 31.85 & 185 & 1123.03 & 8423.84 \\
\hline 35 & 5.63 & 42.21 & 190 & 1254.95 & 9413.36 \\
\hline 40 & 7.38 & 55.37 & 195 & 1398.32 & 10488.76 \\
\hline 45 & 9.59 & 71.93 & 200 & 1554.35 & 11659.16 \\
\hline 50 & 12.34 & 92.59 & 205 & 1722.90 & 12923.45 \\
\hline 60 & 19.93 & 149.51 & 210 & 1906.20 & 14298.38 \\
\hline
\end{tabular}




\section{CHECK COPY}

\begin{tabular}{|c|c|c|c|c|c|}
\hline Temp $^{\circ} \mathrm{C}$ & $\mathrm{kPa}$ & torr & Temp $^{\circ} \mathrm{C}$ & $\mathrm{kPa}$ & torr $^{*}$ \\
\hline 65 & 25.02 & 187.69 & 215 & 2104.20 & 15783.58 \\
\hline 70 & 31.18 & 233.85 & 220 & 2317.80 & 17385.79 \\
\hline 75 & 38.56 & 289.26 & 225 & 2547.90 & 19111.76 \\
\hline 80 & 47.37 & 355.34 & 230 & 2795.10 & 20966.01 \\
\hline 90 & 57.82 & 433.67 & 235 & 3060.40 & 22956.02 \\
\hline 95 & 70.12 & 525.95 & 240 & 3344.70 & 25088.55 \\
\hline 100 & 84.53 & 634.05 & 245 & 3648.80 & 27369.60 \\
\hline 105 & 101.32 & 760.00 & 250 & 3973.60 & 29805.92 \\
\hline 110 & 120.79 & 906.04 & 255 & 4320.20 & 32405.76 \\
\hline 115 & 143.24 & 1074.44 & 260 & 4689.40 & 35175.13 \\
\hline 120 & 169.02 & 1267.82 & 265 & 5082.30 & 38122.27 \\
\hline 125 & 198.48 & 1488.80 & 270 & 5499.90 & 41254.68 \\
\hline 130 & 232.01 & 1740.30 & 275 & 5943.10 & 44579.12 \\
\hline 135 & 270.02 & 2025.42 & 280 & 6413.20 & 48105.33 \\
\hline 140 & 312.93 & 2347.28 & 285 & 6911.10 & 51840.07 \\
\hline 145 & 361.19 & 2709.28 & 290 & 7438.00 & 55792.34 \\
\hline 150 & 415.51 & 3116.76 & 295 & 7995.20 & 59971.89 \\
\hline & 475.72 & 3568.37 & 300 & 8583.80 & 64386.97 \\
\hline
\end{tabular}

\subsubsection{Density of Water as a Function of Temperature}

Table 4-3 lists the density (gm/liter) of water as a function of temperature. The data were taken from Perry and Chilton (1973).

Table 4-3. Density of Pure Water as a Function of Temperature (Perry and Chilton 1973)

\begin{tabular}{|c|c|c|c|c|c|c|c|}
\hline $\begin{array}{c}\text { Temp } \\
\left({ }^{\circ} \mathrm{C}\right)\end{array}$ & $\begin{array}{c}\text { Density } \\
(\mathbf{g m} / \text { liter })\end{array}$ & $\begin{array}{c}\text { Temp } \\
\left({ }^{\circ} \mathrm{C}\right)\end{array}$ & $\begin{array}{c}\text { Density } \\
(\mathbf{g m} / \text { /iter })\end{array}$ & $\begin{array}{c}\text { Temp } \\
\left({ }^{\circ} \mathrm{C}\right)\end{array}$ & $\begin{array}{c}\text { Density } \\
(\mathbf{g m} / \text { /iter })\end{array}$ & $\begin{array}{c}\text { Temp } \\
\left({ }^{\circ} \mathrm{C}\right)\end{array}$ & $\begin{array}{c}\text { Density } \\
(\mathbf{g m} / \text { liter })\end{array}$ \\
\hline 0 & 0.99987 & 24 & 0.99733 & 48 & 0.98896 & 180 & 0.8866 \\
\hline 1 & 0.99993 & 25 & 0.99708 & 49 & 0.98852 & 190 & 0.8750 \\
\hline 2 & 0.99997 & 26 & 0.99682 & 50 & 0.98807 & 200 & 0.8628 \\
\hline 3 & 0.99999 & 27 & 0.99655 & 51 & 0.98762 & 210 & 0.850 \\
\hline 4 & 1.00000 & 28 & 0.99627 & 52 & 0.98715 & 220 & 0.837 \\
\hline 5 & 0.99999 & 29 & 0.99598 & 53 & 0.98669 & 230 & 0.823 \\
\hline 6 & 0.99997 & 30 & 0.99568 & 54 & 0.98621 & 240 & 0.809 \\
\hline 7 & 0.99993 & 31 & 0.99537 & 55 & 0.98573 & 250 & 0.794 \\
\hline 8 & 0.99988 & 32 & 0.99506 & 60 & 0.98324 & & \\
\hline 9 & 0.99981 & 33 & 0.99473 & 65 & 0.98059 & & \\
\hline 10 & 0.99973 & 34 & 0.99440 & 70 & 0.97781 & & \\
\hline 11 & 0.99963 & 35 & 0.99406 & 75 & 0.977489 & & \\
\hline 12 & 0.99952 & 36 & 0.99371 & 80 & 0.97183 & & \\
\hline 13 & 0.99940 & 37 & 0.99336 & 85 & 0.96865 & & \\
\hline 14 & 0.99927 & 38 & 0.99300 & 90 & 0.96534 & & \\
\hline 15 & 0.99913 & 39 & 0.99263 & 95 & 0.96192 & & \\
\hline 16 & 0.99897 & 40 & 0.99225 & 100 & 0.95838 & & \\
\hline 17 & 0.99880 & 41 & 0.99187 & 110 & 0.9510 & & \\
\hline 18 & 0.99862 & 42 & 0.99147 & 120 & 0.9434 & & \\
\hline 19 & 0.99843 & 43 & 0.99107 & 130 & 0.9352 & & \\
\hline
\end{tabular}


CHECK COPY

\begin{tabular}{|c|c|c|c|c|c|c|c|}
\hline $\begin{array}{c}\text { Temp } \\
\left({ }^{\circ} \mathrm{C}\right)\end{array}$ & $\begin{array}{c}\text { Density } \\
(\mathrm{gm} / \text { liter })\end{array}$ & $\begin{array}{c}\text { Temp } \\
\left({ }^{\circ} \mathrm{C}\right)\end{array}$ & $\begin{array}{c}\text { Density } \\
(\mathbf{g m} / \text { liter })\end{array}$ & $\begin{array}{c}\text { Temp } \\
\left({ }^{\circ} \mathrm{C}\right)\end{array}$ & $\begin{array}{c}\text { Density } \\
(\mathrm{gm} / \text { /lter })\end{array}$ & $\begin{array}{c}\text { Temp } \\
\left({ }^{\circ} \mathrm{C}\right)\end{array}$ & $\begin{array}{c}\text { Density } \\
(\mathrm{gm} / \text { liter })\end{array}$ \\
\hline 20 & 0.99823 & 44 & 0.99066 & 140 & 0.9264 & & \\
\hline 21 & 0.99802 & 45 & 0.99025 & 150 & 0.9173 & & \\
\hline 22 & 0.99780 & 46 & 0.98982 & 160 & 0.9075 & & \\
\hline 23 & 0.99757 & 47 & 0.98940 & 170 & 0.8973 & & \\
\hline
\end{tabular}

\subsubsection{Surface Tension of Pure Water as a Function of Temperature}

Table 4-4 lists the surface tension of pure water in contact with air (Weast and Astle 1981).

Table 4-4. Surface Tension of Pure Water as a Function of Temperature (Weast and Astle 1981)

\begin{tabular}{|c|c|c|c|}
\hline $\begin{array}{c}\text { Temperature } \\
\left({ }^{\circ} \mathrm{C} \text { ) }\right.\end{array}$ & $\begin{array}{c}\text { Surface Tension } \\
\text { (dynes/cm) }\end{array}$ & $\begin{array}{c}\text { Surface Tension } \\
\text { (joules } / \mathbf{m}^{2} \text { ) }\end{array}$ & $\begin{array}{c}\text { Surface Tension } \\
\text { (dynes } / \mathrm{cm} \text { ) }\end{array}$ \\
\hline 20 & 72.75 & 0.07275 & 72.75 \\
\hline 25 & 71.97 & 0.07197 & 71.97 \\
\hline 30 & 71.18 & 0.07118 & 71.18 \\
\hline 40 & 69.56 & 0.06956 & 69.56 \\
\hline 50 & 67.91 & 0.06791 & 67.91 \\
\hline 60 & 66.18 & 0.06618 & 66.18 \\
\hline 70 & 64.40 & 0.06440 & 64.40 \\
\hline 80 & 62.60 & 0.06260 & 62.60 \\
\hline 100 & 58.90 & 0.05890 & 58.90 \\
\hline
\end{tabular}

\subsubsection{Atomic and Molecular Weights of Various Elements \& Molecules}

Table 4-5 is a list of the atomic weights of various elements and the molecular weights of various species (Weast and Astle 1981).

Table 4-5. Listing of the Atomic and Molecular Weights of Elements and Molecules Relevant to the Constituents in Yucca Mountain Waters

\begin{tabular}{|l|c|l|c|}
\hline Element & $\begin{array}{c}\text { Atomic weight } \\
\text { (gm/gm-mol) }\end{array}$ & \multicolumn{1}{|c|}{ Molecule } & $\begin{array}{c}\text { Molecular Weight } \\
\text { (gm/gm-mol) }\end{array}$ \\
\hline $\mathrm{H}$ & 1.0079 & $\mathrm{CaCO}_{3}$ & 100.09 \\
\hline $\mathrm{Na}$ & 22.98977 & $\mathrm{CaCl}$ & 110.99 \\
\hline $\mathrm{K}$ & 39.0983 & $\mathrm{Ca}(\mathrm{OH})_{2}$ & 74.09 \\
\hline $\mathrm{Mg}$ & 24.305 & $\mathrm{CaF}$ & 78.08 \\
\hline $\mathrm{Ca}$ & 40.08 & $\mathrm{CaSO}_{4}$ & 136.14 \\
\hline $\mathrm{C}$ & 12.011 & $\mathrm{Ca}\left(\mathrm{NO}_{3}\right)_{2}$ & 164.09 \\
\hline $\mathrm{Si}$ & 28.0855 & $\mathrm{H}_{2} \mathrm{O}$ & 18.0153 \\
\hline $\mathrm{N}$ & 14.0067 & $\mathrm{MgCO}_{3}$ & 84.32 \\
\hline $\mathrm{O}$ & 15.9994 & $\mathrm{MgCl}_{2}$ & 95.22 \\
\hline $\mathrm{S}$ & 32.06 & $\mathrm{MgF}_{2}$ & 62.31 \\
\hline $\mathrm{F}$ & 18.998403 & $\mathrm{Mg}$ & 184.35 \\
\hline $\mathrm{Cl}$ & 35.435 & $\left.\mathrm{MgSO}_{3}\right)_{2} \cdot 2 \mathrm{H}_{2} \mathrm{O}$ & 120.37 \\
\hline & & $\mathrm{K}_{2} \mathrm{CO}_{3}$ & 138.21 \\
\hline
\end{tabular}


CHECK COPY

\begin{tabular}{|l|l|l|c|}
\hline Element & \multicolumn{1}{|c|}{$\begin{array}{c}\text { Atomic weight } \\
\text { (gm/gm-mol) }\end{array}$} & $\begin{array}{c}\text { Molecule } \\
\text { (gm/gm-mol) }\end{array}$ \\
\hline & & $\mathrm{KHCO}_{3}$ & 100.12 \\
\hline & $\mathrm{KCl}$ & 74.56 \\
\hline & $\mathrm{KF}$ & 58.10 \\
\hline & & $\mathrm{KNO}_{3}$ & 101.11 \\
\hline & & $\mathrm{K}_{2} \mathrm{SO}_{4}$ & 174.27 \\
\hline & & $\mathrm{Na}_{2} \mathrm{CO}_{3}$ & 105.99 \\
\hline & & $\mathrm{NaHCO}_{3}$ & 84.00 \\
\hline & & $\mathrm{NaCl}$ & 58.44 \\
\hline & & $\mathrm{NaF}$ & 41.99 \\
\hline & & $\mathrm{NaNO}_{3}$ & 84.99 \\
\hline & & $\mathrm{Na}_{2} \mathrm{SO}_{4}$ & 142.04 \\
\hline & & $\mathrm{O}_{2}$ & 31.9988 \\
\hline
\end{tabular}

\subsubsection{Equilibrium Constant for Water Dissociation, $K_{W}$}

Table 4-6 lists the equilibrium constant for water dissociation, $K_{W}$, as a function of temperature (Weast and Astle 1981).

Table 4-6. Equilibrium Water Dissociation Constant as a Function of Temperature

\begin{tabular}{|c|c|}
\hline Temperature $\left({ }^{\circ} \mathrm{C}\right)$ & $-\log \left(K_{W}\right)$ \\
\hline 0 & 14.9435 \\
\hline 5 & 14.7338 \\
\hline 10 & 14.5346 \\
\hline 15 & 14.3463 \\
\hline 20 & 14.1669 \\
\hline 24 & 14.0000 \\
\hline 25 & 13.9965 \\
\hline 30 & 13.8330 \\
\hline 35 & 13.6801 \\
\hline 40 & 13.5348 \\
\hline 45 & 13.3960 \\
\hline 50 & 13.2617 \\
\hline 55 & 13.1369 \\
\hline 60 & 13.0171 \\
\hline
\end{tabular}




\subsubsection{Boiling Points of Saturated Solutions of Some Pure Salts}

Table 4-7 lists the boiling points of several aqueous solutions of pure salts.

Table 4-7. Boiling Points of Aqueous Solutions of Pure Salts (Frazier 1928)

\begin{tabular}{|l|l|}
\hline \multicolumn{1}{|c|}{ Salt } & Boiling point $\left({ }^{\circ} \mathrm{C}\right)$ \\
\hline $\mathrm{K}_{2} \mathrm{SO}_{4}$ & 102 \\
\hline $\mathrm{KCl}$ & 108.6 \\
\hline $\mathrm{NaCl}$ & 109 \\
\hline $\mathrm{NaNO}_{3}$ & 120.6 \\
\hline
\end{tabular}

\subsubsection{Equilibrium Relative Humidity of Selected Saturated Salt Solutions from 0 to $100^{\circ} \mathrm{C}$}

Table 4-8 lists the equilibrium relative humidities of selected saturated salt solutions (Greenspain 1977). These equilibrium relative humidities are also called the deliquescence points. The deliquescence points may also be described as the relative humidities at which these salts will begin sorbing water from the atmosphere.

\subsubsection{Solubilities of $\mathrm{Ca}, \mathrm{Mg}, \mathrm{Na}$, and $\mathrm{K}$ Salts/Minerals in Water}

Table 4-9 is a list of solubilities of the salts/minerals of calcium, magnesium, sodium, and potassium (Weast and Astle 1981). 


\section{CHECK COPY}

Table 4-8. Equilibrium Relative Humidity of Saturated Aqueous Solutions of Various Pure Salts

\begin{tabular}{|c|c|c|c|c|c|c|c|c|c|c|c|}
\hline $\begin{array}{c}\text { Temp } \\
\left({ }^{\circ} \mathrm{C}\right)\end{array}$ & $K F$ & $\mathrm{MgCl}_{2}$ & $\mathrm{~K}_{2} \mathrm{CO}_{3}$ & $\mathrm{MgNO}_{3}$ & $\mathrm{NaNO}_{3}$ & $\mathrm{NaCl}$ & $\mathrm{KCl}$ & $\mathrm{KNO}_{3}$ & $\mathrm{NaOH}$ & $\mathrm{KOH}$ & $\mathrm{K}_{2} \mathrm{SO}_{4}$ \\
\hline 0 & & 33.66 & 43.13 & 60.35 & & 75.51 & 88.61 & 96.33 & & & 98.77 \\
\hline 5 & & 33.60 & 43.13 & 58.86 & 78.57 & 75.65 & 87.67 & 96.27 & & 14.34 & 98.48 \\
\hline 10 & & 33.47 & 43.14 & 57.36 & 77.53 & 75.67 & 86.77 & 95.96 & & 12.34 & 98.18 \\
\hline 15 & & 33.30 & 43.15 & 55.87 & 76.46 & 75.61 & 85.92 & 95.41 & 9.57 & 10.68 & 97.89 \\
\hline 20 & & 33.07 & 43.15 & 54.38 & 75.36 & 75.47 & 85.11 & 94.62 & 8.91 & 9.32 & 97.59 \\
\hline 25 & 30.85 & 32.78 & 43.16 & 52.89 & 74.25 & 75.29 & 84.34 & 93.58 & 8.24 & 8.23 & 97.30 \\
\hline 30 & 27.27 & 32.44 & 43.17 & 51.40 & 73.14 & 75.09 & 83.62 & 92.31 & 7.58 & 7.38 & 97.00 \\
\hline 35 & 24.59 & 32.05 & & 49.91 & 72.06 & 74.87 & 82.95 & 90.19 & 6.92 & 6.73 & 96.71 \\
\hline 40 & 22.68 & 31.60 & & 48.42 & 71.00 & 74.68 & 82.32 & 89.03 & 6.26 & 6.26 & 96.41 \\
\hline 45 & 21.40 & 31.10 & & 46.93 & 69.99 & 74.52 & 81.74 & 87.03 & 5.60 & 5.94 & 96.12 \\
\hline 50 & 20.80 & 30.54 & & 45.44 & 69.04 & 74.43 & 81.20 & 84.78 & 4.94 & 5.72 & 95.82 \\
\hline 55 & 20.60 & 29.93 & & & 68.15 & 74.41 & 80.7 & & 4.27 & 5.58 & \\
\hline 60 & 20.77 & 29.26 & & & 67.35 & 74.50 & 80.25 & & 3.61 & 5.49 & \\
\hline 65 & 21.18 & 28.54 & & & 66.64 & 74.71 & 79.85 & & 2.95 & 5.41 & \\
\hline 70 & 21.74 & 27.77 & & & 66.04 & 75.06 & 79.49 & & 2.29 & 5.32 & \\
\hline 75 & 22.33 & 26.94 & & & 65.56 & 75.58 & 79.17 & & 1.63 & & \\
\hline 80 & 22.85 & 26.05 & & & 65.22 & 76.29 & 78.90 & & & & \\
\hline 85 & 23.20 & 25.11 & & & 65.03 & & 78.68 & & & & \\
\hline 90 & 23.27 & 24.12 & & & 65.00 & & 78.50 & & & & \\
\hline 95 & & 23.07 & & & & & & & & & \\
\hline 100 & & 21.97 & & & & & & & & & \\
\hline
\end{tabular}


CHECK COPY

Table 4-9. Solubilities of $\mathrm{Ca}, \mathrm{Mg}, \mathrm{Na}$, and $\mathrm{K}$ Salts/Minerals in Water

\begin{tabular}{|c|c|c|c|c|c|c|c|c|}
\hline \multirow[b]{2}{*}{ Compound } & \multirow[b]{2}{*}{ Formula } & & \multicolumn{3}{|c|}{ Low Temperature } & \multicolumn{3}{|c|}{ High Temperature } \\
\hline & & & $\begin{array}{c}\text { Temp } \\
\left({ }^{\circ} \mathrm{C}\right)\end{array}$ & $\begin{array}{l}\text { Solubility } \\
\text { gms/100cc }\end{array}$ & $\begin{array}{l}\text { Solubility } \\
\text { mg/liter }\end{array}$ & Temp $\left({ }^{\circ} \mathrm{C}\right)$ & $\begin{array}{l}\text { Solubility } \\
\text { gms/100cc }\end{array}$ & $\begin{array}{l}\text { Solubility } \\
\text { mg/liter }\end{array}$ \\
\hline Calcium sulfate & $\mathrm{CaSO}_{4}$ & Natural anhydrite & 30 & 0.2090 & 2090 & & 0.1619 & 1619 \\
\hline $\begin{array}{l}\text { Calcium sulfate } \\
\text { dihydrate }\end{array}$ & $\mathrm{CaSO}_{4} \cdot 2 \mathrm{H}_{2} \mathrm{O}$ & Natural gypsum & & 0.2410 & 2410 & & 0.222 & 2220 \\
\hline Calcium nitrate & $\mathrm{Ca}\left(\mathrm{NO}_{3}\right)_{2}$ & & 18 & 121.2 & 1212000 & & 376 & 3760000 \\
\hline Calcium chloride & $\mathrm{CaCl}_{2}$ & & 20 & 74.5 & 745000 & & 159 & 1590000 \\
\hline Calcium fluoride & $\mathrm{CaF}_{2}$ & Natural fluorite & 18 & 0.0016 & 16 & & 0.0017 & 17 \\
\hline Calcium carbonate & $\mathrm{CaCO}_{3}$ & Calcite & 25 & 0.0014 & 14 & & 0.0018 & 18 \\
\hline Calcium hydroxide & $\mathrm{Ca}(\mathrm{OH})_{2}$ & & 0 & 0.1850 & 1850 & & 0.077 & 770 \\
\hline Sodium Sulfate & $\mathrm{Na}_{2} \mathrm{SO}_{4}$ & Natural thenardite & 0 & 4.760 & 47600 & & 42.7 & 427000 \\
\hline Sodium nitrate & $\mathrm{NaNO}_{3}$ & Soda niter & 25 & 92.1 & 921000 & & 180 & 1800000 \\
\hline Sodium chloride & $\mathrm{NaCl}$ & Halite & 0 & 35.7 & 357000 & & 39.12 & 391200 \\
\hline Sodium fluoride & $\mathrm{NaF}$ & Natural villiaumite & 18 & 4.22 & 42200 & & & 0 \\
\hline Sodium carbonate & $\mathrm{Na}_{2} \mathrm{CO}_{3}$ & & 0 & 7.1 & 71000 & & 45.5 & 455000 \\
\hline $\begin{array}{l}\text { Sodium } \\
\text { bicarbonate }\end{array}$ & $\mathrm{NaHCO}_{3}$ & & 0 & 6.9 & 69000 & & 16.4 & 164000 \\
\hline Sodium hydroxide & $\mathrm{NaOH}$ & & 0 & 42.0 & 420000 & & 347 & 3470000 \\
\hline Sodium silicate & $\mathrm{Na}_{2} \mathrm{SiO}_{3}$ & Metasilicate & & Soluble & & & Dissolves & \\
\hline Magnesium sulfate & $\mathrm{MgSO}_{4}$ & & 0 & 26 & 260000 & & 73.8 & 738000 \\
\hline Magnesium nitrate & $\mathrm{Mg}\left(\mathrm{NO}_{3}\right)_{2}$ & & & 124 & 1240000 & & Vs & \\
\hline $\begin{array}{l}\text { Magneslum } \\
\text { chloride }\end{array}$ & $\mathrm{MgCl}_{2}$ & & 20 & 54.25 & 542500 & & 72.7 & 727000 \\
\hline $\begin{array}{l}\text { Magnesium } \\
\text { fluoride }\end{array}$ & $\mathrm{MgF}_{2}$ & Natural sellaite & 18 & 0.0076 & 76 & & Insoluble & \\
\hline $\begin{array}{l}\text { Magnesium } \\
\text { carbonate }\end{array}$ & $\mathrm{MgCO}_{3}$ & Natural magnesite & & 0.0106 & 106 & & & \\
\hline $\begin{array}{l}\text { Magnesium } \\
\text { carbonate } \\
\text { trihydrate }\end{array}$ & $\mathrm{MgCO}_{3} \cdot 3 \mathrm{H}_{2} \mathrm{O}$ & Natural hydromag & & 0.04 & 400 & & 0.011 & 110 \\
\hline $\begin{array}{l}\text { Magnesium } \\
\text { carbonate basic }\end{array}$ & $3 \mathrm{MgCO}_{3} \cdot \mathrm{Mg}(\mathrm{OH})_{2}$ & Natural nesqueho & $\overline{16}$ & $0 . \overline{179}$ & 1790 & & Dissolves & \\
\hline $\begin{array}{l}\text { Magnesium } \\
\text { hydroxide }\end{array}$ & $\mathrm{Mg}(\mathrm{OH})_{2}$ & Natural brucite & 18 & 0.0009 & 9 & & 0.004 & 40 \\
\hline
\end{tabular}


CHECK COPY

\begin{tabular}{|c|c|c|c|c|c|c|c|c|}
\hline & & & \multicolumn{3}{|c|}{ Low Temperature } & \multicolumn{3}{|c|}{ High Temperature } \\
\hline Compound & Formula & & $\begin{array}{l}\text { Temp } \\
\left({ }^{\circ} \mathrm{C}\right)\end{array}$ & $\begin{array}{l}\text { Solubility } \\
\text { gms/100cc }\end{array}$ & $\begin{array}{l}\text { Solubility } \\
\text { mg/liter }\end{array}$ & $\operatorname{Temp}\left({ }^{\circ} \mathrm{C}\right)$ & $\begin{array}{c}\text { Solubility } \\
\text { gms/100cc }\end{array}$ & $\begin{array}{l}\text { Solubility } \\
\mathrm{mg} / \text { /liter }\end{array}$ \\
\hline Potassium sulfate & $\mathrm{K}_{2} \mathrm{SO}_{4}$ & Arcanite & 25 & 12 & 120000 & & 24.1 & 241000 \\
\hline $\begin{array}{l}\text { Potassium sulfate, } \\
\text { hydrogen }\end{array}$ & $\mathrm{KHSO}_{4}$ & Mercallite & 0 & 36.3 & 363000 & & 121.6 & 1216000 \\
\hline Potassium nitrate & $\mathrm{KNO}_{3}$ & Saltpeter & 0 & 13.3 & 133000 & & 247 & 2470000 \\
\hline $\begin{array}{l}\text { Potassium } \\
\text { chloride }\end{array}$ & $\mathrm{KCl}$ & Sylvite & 20 & 23.8 & 238000 & & 56.7 & 567000 \\
\hline Potassium fluoride & $\mathrm{KF}$ & & 18 & 92.3 & 923000 & & Very soluble & \\
\hline $\begin{array}{l}\text { Potassium } \\
\text { carbonate }\end{array}$ & $\mathrm{K}_{2} \mathrm{CO}_{3}$ & & 20 & 112 & 1120000 & & 156 & 1560000 \\
\hline $\begin{array}{l}\text { Potassium } \\
\text { carbonate, } \\
\text { hydrogen }\end{array}$ & $\mathrm{KHCO}_{3}$ & & & 22.4 & 224000 & & 60 & 600000 \\
\hline $\begin{array}{l}\text { Potassium } \\
\text { hydroxide }\end{array}$ & $\mathrm{KOH}$ & & 15 & & & & 178 & 1780000 \\
\hline
\end{tabular}




\section{CHECK COPY}

\subsubsection{Decomposition/Melting Temperatures of Some Bicarbonate/Carbonate Species}

Table 4-10 is list of the decomposition/melting temperatures of relevant bicarbonate/carbonate species (Weast and Astle 1981).

Table 4-10. Decomposition/Melting Temperatures of Relevant Bicarbonate/Carbonate Species

\begin{tabular}{|c|c|c|l|}
\hline Species & $\begin{array}{c}\text { Decomposition } \\
\text { Temp }\left({ }^{\circ} \mathrm{C}\right)\end{array}$ & Melting Temp $\left({ }^{\circ} \mathrm{C}\right)$ & \multicolumn{1}{|c|}{$\begin{array}{c}\text { Decomposition } \\
\text { Product }\end{array}$} \\
\hline $\mathrm{KHCO}_{3}$ & 100 & $\mathrm{~N} / \mathrm{A}$ & $\mathrm{CO}, \mathrm{CO}_{2}, \mathrm{KOH}, \mathrm{K} 2 \mathrm{O}$ \\
\hline $\mathrm{NaHCO}_{3}$ & 250 & $\mathrm{~N} / \mathrm{A}$ & $\mathrm{CO}_{2}, \mathrm{NaOH}$ \\
\hline
\end{tabular}

\subsubsection{Adsorbed Water Films as a Function of Relative Humidity and Temperature}

Figures 4-1 and 4-2 show the amount of water adsorbed on a nickel as a function of temperature $\left(20\right.$ to $80^{\circ} \mathrm{C}$ ) and relative humidity (20 to $98 \% \mathrm{RH}$ ) (Lee and Staehle 1997$)$.

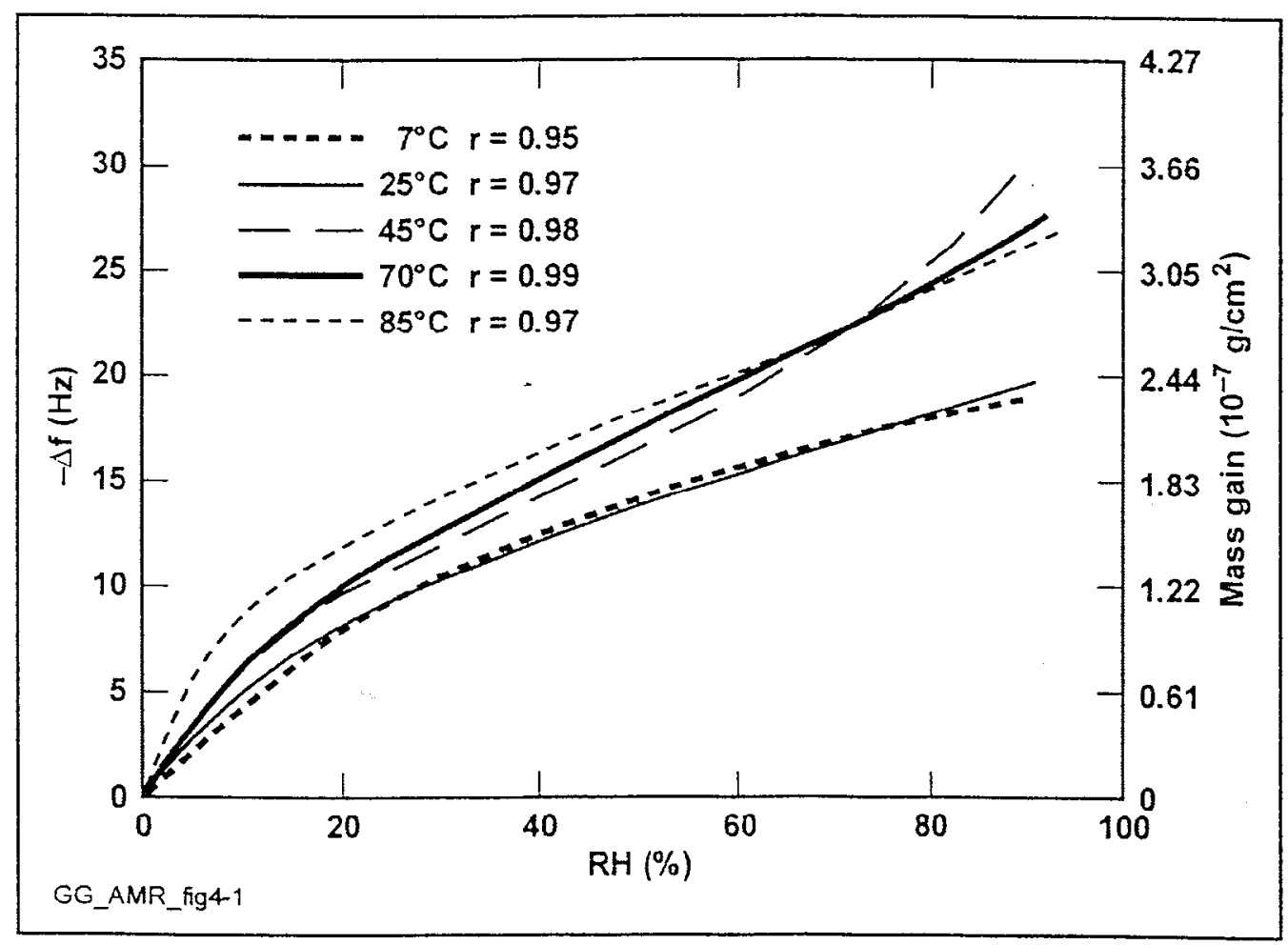

Figure 4-1. Amount of Water Adsorbed on a Nickel as a Function of Temperature $\left(20\right.$ to $\left.80^{\circ} \mathrm{C}\right)$ (Lee and Staehle 1997) 


\section{CHECK COPY}

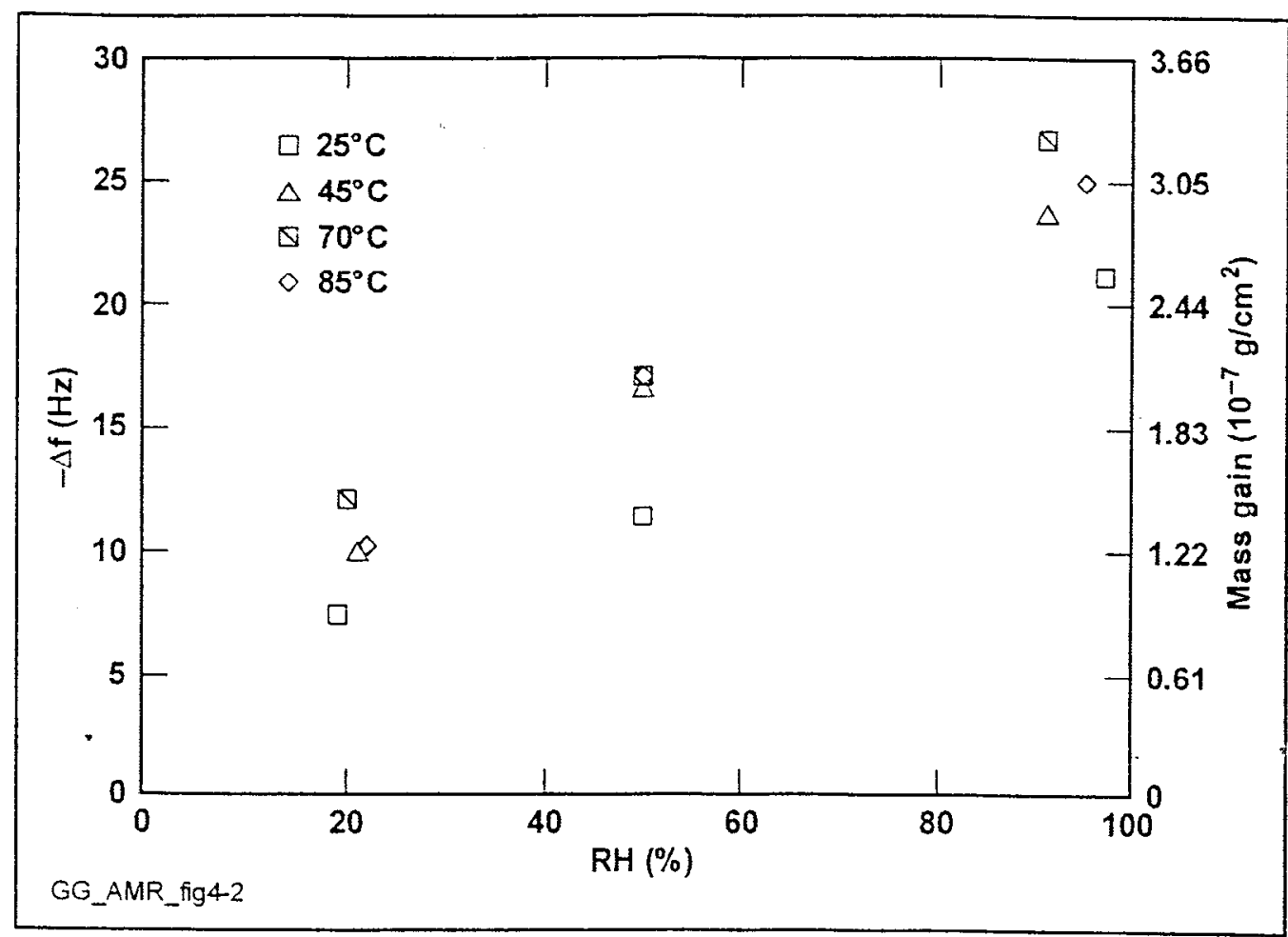

Figure 4-2. Amount of Water Adsorbed on a Nickel as a Function of Relative Humidity (20 to $98 \%$ RH) (Lee and Staehle 1997)

\subsubsection{Vapor Pressure Lowering of Aqueous $\mathrm{NaCl}$ Solutions}

Table 4-11 shows the water vapor pressure lowering of aqueous solutions by $\mathrm{NaCl}$ solutions at temperatures from 20 to $100^{\circ} \mathrm{C}$ (Frazier 1928). The experimental data were reported in terms of two parameters, $m$ and $100 R$, where

$m$ molality (gram-formula weights (moles) of solute per $1000 \mathrm{~g} \mathrm{H}_{2} \mathrm{O}$ )

and

$100 R=100\left(P^{0}-P\right) / m P^{0}$ 
Table 4-11. Experimental Data on the Vapor Pressure Lowering of Aqueous $\mathrm{NaCl}$ Solutions as a Function of Temperature (Frazier 1928)

\begin{tabular}{|c|c|c|c|c|c|}
\hline \multicolumn{2}{|c|}{$20-25^{\circ} \mathrm{C}$} & \multicolumn{2}{c|}{$80^{\circ} \mathrm{C}$} & \multicolumn{2}{c|}{$100^{\circ} \mathrm{C}$} \\
\hline $\mathbf{M}$ & $100 \mathrm{R}$ & $\mathbf{M}$ & $100 \mathrm{R}$ & $\mathbf{M}$ & $100 \mathrm{R}$ \\
\hline 0.1 & 3.29 & 4.0 & 3.65 & 2.5 & 3.47 \\
\hline 0.2 & 3.28 & 5.0 & 3.79 & 3.5 & 3.57 \\
\hline 0.4 & 3.27 & 6.0 & 3.91 & 4.0 & 3.62 \\
\hline 0.6 & 3.27 & & & 5.0 & 3.76 \\
\hline 0.8 & 3.28 & & & 6.3 & 3.91 \\
\hline 1.0 & 3.30 & & & 6.7 & 3.89 \\
\hline 2.0 & 3.42 & & & & \\
\hline 2.8 & 3.53 & & & & \\
\hline 5.0 & 3.87 & & & & \\
\hline 6.0 & 3.99 & & & & \\
\hline
\end{tabular}

From the vapor pressure lowering data of aqueous $\mathrm{NaCl}$ solutions (see Table 4-12), the relative humidity $(R H)$ as a function of concentration (both molality and ppm) is calculated.

$100 R=100\left(P^{0}-P\right) / m P^{0}$

where

$P^{0} \quad$ vapor pressure of pure solvent (water)

$P \quad$ partial vapor pressure of the solvent (water) from the solution.

Rearranging the above equation in terms of $P$ gives

$P=P^{0}-m \times P^{0} \times(100 R / 100)$.

The relative humidity $(R H)$ is calculated by the following equation:

$R H=P / P^{0}$

From the molality data the concentration in terms of ppm is calculated from the following equation:

$C(p p m)=m \times M W /\left(1000 g_{H 2 O}+m \times M W\right)$

where

$\mathrm{MW}=$ molecular weight of solute $=(55.44 \mathrm{gm} / \mathrm{mole}$ of $\mathrm{NaCl})$. 


\section{CHECK COPY}

Table 4-12. NaCl Molality as a Function of Temperature

\begin{tabular}{|l|c|c|c|c|c|}
\hline $\begin{array}{c}\text { Temperature } \\
\left({ }^{\circ} \mathrm{C}\right)\end{array}$ & M (molality) & 100R & $\begin{array}{c}\text { Concentration } \\
(\mathrm{ppm})\end{array}$ & $\mathbf{P}(\mathrm{kPa})$ & $\mathbf{R H}(\%)$ \\
\hline $20-25$ & 0.1 & 3.29 & 5810 & 3.16 & 99.7 \\
\hline$\left(\mathrm{P}^{0}=3.17 \mathrm{kPa}\right)$ & 0.2 & 3.28 & 11553 & 3.15 & 99.3 \\
\hline & 0.4 & 3.27 & 22842 & 3.13 & 98.7 \\
\hline & 0.6 & 3.27 & 33876 & 3.10 & 98.0 \\
\hline & 0.8 & 3.28 & 44664 & 3.08 & 97.4 \\
\hline & 1.0 & 3.30 & 55213 & 3.06 & 96.7 \\
\hline & 2.0 & 3.42 & 104649 & 2.95 & 93.2 \\
\hline & 2.8 & 3.53 & 140622 & 2.85 & 90.1 \\
\hline & 5.0 & 3.87 & 226126 & 2.55 & 80.7 \\
\hline & 6.0 & 3.99 & 259610 & 2.41 & 76.1 \\
\hline 80 & & & & & \\
\hline$\left(\mathrm{P}^{0}=47.34 \mathrm{kPa}\right)$ & 4.0 & 3.65 & 189470 & 40.43 & 85.4 \\
\hline & 5.0 & 3.79 & 226126 & 38.37 & 81.1 \\
\hline & 6.0 & 3.91 & 259610 & 36.23 & 76.5 \\
\hline 100 & & & & & \\
\hline $\mathrm{P}^{0}=101.32 \mathrm{kPa}$ & 2.5 & 3.47 & 127476 & 92.53 & 91.3 \\
\hline & 3.5 & 3.57 & 169808 & 88.66 & 87.5 \\
\hline & 4.0 & 3.62 & 189470 & 86.65 & 85.5 \\
\hline & 5.0 & 3.76 & 226126 & 82.27 & 81.2 \\
\hline & 6.3 & 3.91 & 269098 & 76.36 & 75.4 \\
\hline & 6.7 & 3.89 & 281376 & 74.91 & 73.9 \\
\hline
\end{tabular}

\subsubsection{Composition of Well J13 Water}

Table 4-13 lists the composition of well J13 water (Harrar 1990).

Table 4-13. Composition of Well J13 Water (Harrar 1990)

\begin{tabular}{|c|c|}
\hline Species & Concentration (mg/) \\
\hline $\mathrm{Na}$ & 45.80 \\
\hline $\mathrm{Si}$ & 28.5 \\
\hline $\mathrm{Ca}$ & 13.0 \\
\hline $\mathrm{Kg}$ & 5.04 \\
\hline $\mathrm{F}$ & 2.01 \\
\hline $\mathrm{Cl}$ & 2.18 \\
\hline $\mathrm{NO}_{3}$ & 7.14 \\
\hline $\mathrm{SO}_{4}$ & 8.78 \\
\hline $\mathrm{HCO}_{3}$ & 18.4 \\
\hline & 128.9 \\
\hline $\mathrm{pH}$ & \\
\hline
\end{tabular}




\section{CHECK COPY}

\subsubsection{Composition of Waters from Field Studies (TBD)}

The composition of waters that have been obtained from the Single Heater Test. and the Drift Scale Test field studies at Yucca Mountain will be noted in this section.

\subsubsection{Compositions of Perched and Pore Waters from Yucca Mountain (TBD)}

The compositions of waters that have been obtained from perched and pore waters from Yucca Mountain will be noted in this section.

\subsubsection{Compositions of Waters Contacting the Drip Shield and Waste Package Outer Barrier Surfaces (TBD)}

When the analyses from N3080 "Description Abstract NFE In-Drift Water," EB225 "In-Drift THC Analysis," EB45 "Precipitate Salts Analysis," and possibly EB165 "Seepage/Backfill Interaction" have been completed, the results will be analyzed in the context of this $3.10 \mathrm{Q}$ AMR.

\subsubsection{Henry's Law Constants for the Solubility of Oxygen in Several Aqueous Solutions}

Table 4-14 lists parameters for calculating the Henry's Law Constant for oxygen solubility in pure water and three aqueous $\mathrm{NaCl}$ solutions (Cramer 1980). The Henry's Law constant, $k$, was determined experimentally, and the variation with temperature fit to the following equation.

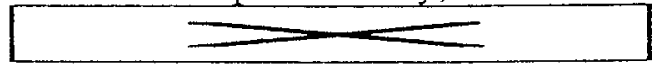

$\ln k=a_{0}+a_{1} / T+a_{2} / T^{2}+a_{3} / T^{3}+a_{4} / T^{4}$

where $a_{0}, a_{1}, a_{3}$, and $a_{4}$, are experimentally determined constants, and $T$ is temperature in Kelvin.

Also included in Table 4-14 are the characteristics of the fluids for which the $\mathrm{k}$ values were determined.

Tabie 4-14. Coefficients for Henry's Law Constants for the Oxygen Solubility in the $\mathrm{NaCl}$ Solutions and Demineralized Water

\begin{tabular}{|l|c|c|c|c|c|c|c|c|}
\hline Solution & Molality & Molarity & \multicolumn{7}{|c|}{ Coefficients } \\
\hline & $\begin{array}{c}\text { (moles/ } \\
1,000 \mathrm{~g})\end{array}$ & $\begin{array}{c}\text { (moles/ } \\
\text { liter) }\end{array}$ & $\mathbf{a}_{0}$ & $\mathbf{a}_{1}\left(10^{4}\right)$ & $\mathbf{a}_{2}\left(10^{7}\right)$ & $\mathbf{a}_{3}\left(10^{9}\right)$ & $\mathbf{a}_{4}\left(10^{11}\right)$ & $\begin{array}{c}\text { Std dev, } \\
\%\end{array}$ \\
\hline $\begin{array}{l}\text { Deminer- } \\
\text { alized water }\end{array}$ & 0 & 0 & -35.4408 & 5.58970 & -2.67211 & 5.80947 & -4.91667 & 5.6 \\
\hline $\mathrm{NaCl}-1$ & 0.87 & 0.85 & -23.8312 & 4.05434 & -1.89188 & 4.02544 & -3.35511 & 4.5 \\
\hline $\mathrm{NaCl}-2$ & 2.97 & 2.78 & -14.7705 & 2.95905 & -1.38197 & 2.97589 & -2.51663 & 5.3 \\
\hline $\mathrm{NaCl}-3$ & 5.69 & 5.09 & 18.7594 & -2.02638 & 1.38823 & -3.75034 & 3.52602 & 4.9 \\
\hline
\end{tabular}

Table 4-15 lists the Henry's Law constant that was calculated using the coefficients in Table 4-14. 


\section{CHECK COPY}

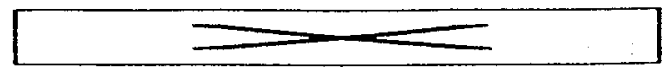

$\ln k=a_{0}+a_{1} / T+a_{2} / T^{2}+a_{3} / T^{3}+a_{4} / T^{4}$

Table 4-15. Calculated Henry's Law Constants for Oxygen Solubility in Three Aqueous Solutions

\begin{tabular}{|c|c|c|c|c|}
\hline \multirow[b]{2}{*}{ Temp $\left({ }^{\circ} \mathrm{C}\right)$} & \multicolumn{4}{|c|}{$\begin{array}{c}\text { Henry's Law Constants, } k \\
\text { MPa }\end{array}$} \\
\hline & $\begin{array}{c}\text { Demineralized } \\
\text { Water }\end{array}$ & $\mathrm{NaCl}-1$ & $\mathrm{NaCl}-2$ & $\mathrm{NaCl}-3$ \\
\hline 0 & 2427.81 & 3948.40 & 9404.30 & 21073.80 \\
\hline 5 & 2863.54 & 4464.37 & 10208.99 & 21453.07 \\
\hline 10 & 3297.81 & 4963.39 & 10938.31 & 21966.03 \\
\hline 15 & 3722.91 & 5440.21 & 11591.48 & 22565.62 \\
\hline 20 & 4132.95 & 5891.19 & 12170.10 & 23213.24 \\
\hline 25 & 4523.66 & 6313.95 & 12677.33 & 23876.50 \\
\hline 30 & 4892.07 & 6707.11 & 13117.18 & 24528.06 \\
\hline 35 & 5236.23 & 7069.92 & 13494.07 & 25144.91 \\
\hline 40 & 5554.97 & 7402.14 & 13812.51 & 25708.11 \\
\hline 45 & 5847.63 & 7703.84 & 14076.89 & 26202.50 \\
\hline 50 & 6113.91 & 7975.28 & 14291.32 & 26616.56 \\
\hline 55 & 6353.75 & 8216.84 & 14459.60 & 26942.21 \\
\hline 60 & 6567.23 & 8428.99 & 14585.22 & 27174.52 \\
\hline 65 & 6754.52 & 8612.21 & 14671.31 & 27311.44 \\
\hline 70 & 6915.85 & 8767.04 & 14720.70 & 27353.44 \\
\hline 75 & 7051.50 & 8894.05 & 14735.98 & 27303.16 \\
\hline 80 & 7161.78 & 8993.82 & 14719.45 & 27165.00 \\
\hline 85 & 7247.07 & 9067.00 & 14673.26 & 26944.75 \\
\hline 90 & 7307.78 & 9114.26 & 14599.38 & 26649.23 \\
\hline 95 & 7344.41 & 9136.37 & 14499.63 & 26285.95 \\
\hline 100 & 7357.55 & 9134.14 & 14375.74 & 25862.82 \\
\hline 105 & 7347.86 & 9108.45 & 14229.36 & 25387.88 \\
\hline 110 & 7316.12 & 9060.26 & 14062.06 & 24869.08 \\
\hline 115 & 7263.18 & 8990.61 & 13875.36 & 24314.14 \\
\hline 120 & 7190.02 & 8900.61 & 13670.75 & 23730.39 \\
\hline 125 & 7097.72 & 8791.44 & 13449.69 & 23124.66 \\
\hline 130 & 6987.44 & 8664.34 & 13213.58 & 22503.24 \\
\hline 135 & 6860.44 & 8520.59 & 12963.04 & 21871.86 \\
\hline 140 & 6718.03 & 8361.53 & 12701.82 & 21235.62 \\
\hline
\end{tabular}

\subsubsection{Evaporative/Boiling Concentrating of a Simulated Well J13 Water Near the Boiling Point of the Concentrated Water (DTN \#LL990703005924.083 and TBD\#)}

Table 4-16 lists the composition of waters that result from the evaporative/boiling concentrating of simulated concentrated well J13 water. The initial waters are nominally 100 times the concentration of well J13 waters. Samples were measured after concentrating 1 liter, 2 liters, etc. of solution to approximately 100 to $200 \mathrm{ml}$. The water was dripped onto and drained through 


\section{CHECK COPY}

heated crushed tuff. Heat was supplied to the system in order to keep the temperature of the water at or near its boiling point.

The testing is continuing in order to obtain the compositions of aqueous solutions that result from progressively more evaporative concentrating, and also to obtain the boiling point and $\mathrm{pH}$ of the resulting aqueous solutions.

Table 4-16. The Compositions of Evaporatively Concentrated Simulated 100x Well J13 Water

\begin{tabular}{|c|c|c|c|c|c|c|c|c|}
\hline \multirow[t]{2}{*}{ Species } & \multirow[t]{2}{*}{$\begin{array}{c}\text { Starting } \\
\text { Sol'n } \\
\text { (mg/liter) }\end{array}$} & \multicolumn{7}{|c|}{$\begin{array}{l}\text { Solution Compositions (mg/liter) } \\
\text { Amount of Initial Solution Concentrated to } 100-200 \mathrm{ml} \\
\text { and the Estimated Boiling Point }\left({ }^{\circ} \mathrm{C}\right)\end{array}$} \\
\hline & & 1 & 2 & 3 & 4 & 5 & 6 & 7 \\
\hline Sample \# & $\begin{array}{c}\text { Initial J13 } \\
\text { Batch } 3 \\
\end{array}$ & $\begin{array}{c}\text { ECBP } \\
042399-0 \\
\end{array}$ & $\begin{array}{c}E C B P \\
050699-0\end{array}$ & $\begin{array}{c}E C B P \\
051899-0 \\
\end{array}$ & $\begin{array}{c}\text { ECBP } \\
060199-0 \\
\end{array}$ & $\begin{array}{c}\text { ECBP } \\
061099-0 \\
\end{array}$ & $\begin{array}{c}E C B P \\
061499-0\end{array}$ & $\begin{array}{c}\text { ECBP } \\
061599-0\end{array}$ \\
\hline \multicolumn{9}{|c|}{ DTN \#LL990703005924.083 } \\
\hline $\begin{array}{l}\text { Boiling } \\
\text { Point } \\
\end{array}$ & & 101 & & & & & 108 & \\
\hline $\mathrm{Ca}$ & 6 & 735 & 0 & 0 & $\overrightarrow{48}$ & 0 & 62 & 587 \\
\hline $\mathrm{k}$ & 535 & 3885 & 7985 & 15878 & 21180 & 24510 & 21532 & 8389 \\
\hline $\mathrm{Mg}$ & 0 & 0 & 0 & 0 & 0 & 0 & 0 & 0 \\
\hline $\mathrm{Na}$ & 4360 & 43317 & 106465 & 177301 & 202726 & 226721 & 190711 & 92279 \\
\hline SI & 13 & 7461 & 30875 & 79388 & 118912 & 121020 & 90741 & 42504 \\
\hline$F$ & 210 & 2230 & 4392 & 4393 & 0 & 843 & 0 & 3020 \\
\hline $\mathrm{Cl}$ & 729 & 7446 & 16130 & 32814 & 38125 & 49021 & 49831 & 15715 \\
\hline $\mathrm{NO}_{3}$ & 846 & 9370 & 14905 & 34402 & 40243 & 51165 & 50443 & 15184 \\
\hline $\mathrm{SO}_{4}$ & 1790 & 19326 & 38594 & 53455 & 41150 & 48408 & 32913 & 30480 \\
\hline $\mathrm{HCO}_{3}$ & 9556 & 28921 & 51776 & 107033 & 98361 & 89496 & 79006 & 37750 \\
\hline
\end{tabular}

\subsubsection{Evaporative/Boiling Concentrating of Waters from Field Studies Near the Boiling Point of the Concentrated Water (TBD\#)}

This section will document the compositions, boiling points, and $\mathrm{pH}$ of aqueous solutions that result from the evaporative concentrating of simulated field studies waters, including those from the Single Heater Test and the Drift Scale Test at Yucca Mountain. This analysis will be performed to determine if significant differences exist between the characteristics of these waters and those of simulated well J13 water.

\subsubsection{Evaporative Concentrating of Simulated J13 at Temperatures Below the Boiling Point (DTN \#LL990703005924.083 and TBD\#)}

Table 4-17 characterizes the aqueous solutions that may be in contact with the DSs and the WPOBs in the temperature range in which susceptibility to aqueous electrochemical corrosion is possible. This range includes temperatures between 85 and $110^{\circ} \mathrm{C}$, and relative humidities from 50 to $90 \%$. Tests are now underway to determine the composition and $\mathrm{pH}$ of the aqueous solutions that result from the evaporative concentrating of simulated J13 water in these ranges. 
Table 4-17. Results of the Evaporative Concentration of a Simulated Well J13 Water Under Conditions of Constant Temperature and Relative Humidity

\begin{tabular}{|l|c|c|}
\hline \multicolumn{1}{|c|}{ Species } & $\begin{array}{c}\text { Starting } \\
\text { Sol'n } \\
\text { (mg/liter) }\end{array}$ & $\begin{array}{c}\text { Final Sol'n } \\
85 \% \text { RH/90 } \\
\text { (mg/liter) }\end{array}$ \\
\hline Sample \# & $\begin{array}{c}\text { Initial J13 } \\
\text { Batch 1 }\end{array}$ & J13-01 \\
\hline $\mathrm{DTN}$ \#LL990703005924.083 \\
\hline $\mathrm{Cl}$ & 730 & 15200 \\
\hline $\mathrm{NO}_{3}$ & 732 & 14600 \\
\hline $\mathrm{SO}_{4}$ & 1633 & 30400 \\
\hline $\mathrm{F}$ & 208 & 3500 \\
\hline $\mathrm{HCO}{ }_{3}{ }^{*}$ & 4210 & 11370 \\
\hline $\mathrm{Na}$ & 517 & 79700 \\
\hline $\mathrm{K}$ & 5.48 & 10020 \\
\hline $\mathrm{Ca}$ & 2.04 & 26 \\
\hline $\mathrm{Mg}$ & & 0 \\
\hline
\end{tabular}

\subsubsection{Evaporative Concentrating of Waters from Field Studies at Temperatures Below the Boiling Point (TBD)}

This section will document the compositions, boiling points, and $\mathrm{pH}$ of aqueous solutions that result from the evaporative concentrating of simulated field studies waters, including those from the Single Heater Test and the Drift Scale Test at Yucca Mountain. This analysis will be performed to determine if significant differences exist between the characteristics of these waters and those of simulated well $\mathrm{J} 13$ water.

\subsubsection{Gas Phase Compositions in Drift (TBD)}

The composition of the gas phase has a controlling influence on the water chemistry of thin films. Important parameters include the partial pressure of water vapor $\left(\mathrm{H}_{2} \mathrm{O}\right)$, oxygen $\left(\mathrm{O}_{2}\right)$, and carbon dioxide $\left(\mathrm{CO}_{2}\right)$. The water chemistry can also be affected by the presence of aerosols and dust particles containing soluble salts. Aerosols and dust particles in the atmosphere can contain chlorides, sulfates, and nitrates. Because aerosols and dust particles contain soluble salts they can effect water chemistry if present in sufficient quantities.

For the analyses performed in this $3.10 \mathrm{Q}$, the analysis of the gas phase composition is initially unnecessary. The analyses are performed as a function of water vapor content and temperature.

It is not known if the mechanisms for aerosol formation or dry deposition of dust particles will be operative in the drifts after the waste packages (WPs) have been emplaced. Active ventilation may be a mechanism for depositing salts on the DSs and WPs. Further, the soluble salt content of the "dust" in the drifts has not been characterized. 


\section{CHECK COPY}

\subsection{CRITERIA}

The applicable requirements reproduced below are from Section 1.2, "System Design Criteria" of Uncanistered Spent Nuclear"Fuel Disposal Container System Description Document (CRWMS 1999). For the sake of clarity, the original section numbers appear in parentheses after the text.

\subsection{1 “System Environment Criteria” (Section 1.2.3)}

"The disposal container shall meet all performance requirements during and after exposure to the emplacement drift external environments identified in Table 1-8. (TBD-234)." (Section 1.2.3.1)

Table 4-18. Emplacement Drift External Environment (Table 1-8)

\begin{tabular}{|c|c|c|}
\hline Environment & Range & $\begin{array}{c}\text { Duration/Frequency of } \\
\text { Occurrence }\end{array}$ \\
\hline TBD & TBD & TBD \\
\hline
\end{tabular}

The analyses contained in this AMR characterize the range of environments possible on the surfaces of the DSs and WPOBs as a function of the in-drift environment in order that testing may be conducted to determine if the performance requirements for the WPS and DS will be met.

\subsection{2 "System Performance Criteria" (Section 1.2.1)}

"The disposal container shall be designed such that no more than 1 percent of all waste packages breach during the first 1,000 years after emplacement (breaching is defined as an opening through the wall of the waste package through which advective or diffusive transport of gas or radionuclides can occur)." (Section 1.2.1.1)

"The disposal container shall be designed such that for 10,000 years after permanent closure of the repository the release rate of any radionuclide from all waste packages shall not exceed one part in 100,000 per year of the inventory of that radionuclide calculated to be present at 1,000 years following permanent closure; provided, that this requirement does not apply to any radionuclide which is released at a rate less than 0.1 percent of the calculated total release rate limit. The calculated total release rate limit shall be taken to be one part in 100,000 per year of the inventory of radioactive waste, originally cmplaced in the underground facility, that remains after 1,000 years of radioactive decay." (Section 1.2.1.4)

The analyses contained in this AMR characterize the range of environments possible on the surfaces of the DSs and WPOBs. The environments' conditions will be considered in evaluating the performance of the candidate materials for the DSs and WPOBs to determine if the performance requirements for the WPs and DS listed above will be met.

\subsection{CODES AND STANDARDS}

No codes or standards are currently used in this document. 


\section{CHECK COPY}

\section{ASSUMPTIONS}

The following assumptions were used in Section 6 of this report. The assumptions concern the nature of the water contacting the surfaces of the DSs and the WPOBs, and the expected DS and WPOB temperature and relative humidity histories.

- The composition of well $\mathrm{J} 13$ water is representative of water that will contact the DSs' and WPOBs' surfaces. (TBV)

Information concerning this assumption will be obtained from other 3.10Q AMRs, such as "N3080 Description Abstract NFE In-Drift Water." If another water chemistry is determined to be more appropriate, then sensitivity analyses will be perform to determine the consequences of the different water chemistries.

- The temperatures of the DSs and WPOBs are less than $300^{\circ} \mathrm{C}$. (TBV)

Information concerning this assumption will be obtained from other 3.10Q AMRs, such as "EB225 In-Drift THC Analysis."

- The total pressure in the drifts is limited to the ambient nominal atmospheric pressure (87.67 $\mathrm{kPa}$ ), which corresponds to a pure-water boiling point of $96^{\circ} \mathrm{C}$. (TBV)

Information concerning this assumption will be obtained from other 3.10Q AMRs, such as "EB175 Gas Flux \& Composition."

- The gas phase composition of the drift air exclusive of water is either the same as "atmospheric air" or has a concentration of $\mathrm{CO}_{2}$ that is a factor of three higher. (TBV)

Information concerning this assumption will be obtained from other 3.10Q AMRs, such as "EB175 Gas Flux \& Composition." 



\section{CHECK COPY}

\section{ANALYSIS}

\subsection{INTRODUCTION}

The environments on the drip shield and waste package outer barrier are controlled by the compositions of the waters that contact these components, the temperature $(T)$ of these components, and the effective relative humidity $(R H)$ at these components. Because the composition of the waters that are expected to enter the emplacement drifts (either by seepage flow or by episodic flow) have not been specified, well J13 water was chosen as the referencc water (Harrar 1990).

Section 6.2 discusses the accessible $R H$ for the temperatures of interest at the repository horizon. Section 6.3 discusses the adsorption of water on metal alloys in the absence of hygroscopic salts.

Because the temperatures of the DSs and the WPOBs are higher than those of the surrounding near-field environment, the relative humidity at the DSs and the WPOBs will be lower than that of the surrounding near-field environment. This difference is a result of the water partial pressure in the drift being constant and no higher than the equilibrium water vapor pressure at the temperature of the drift wall. Hence,

$P_{H 2 O}(W P)=P_{H 2 O}(D W)$, but

$P_{H 2 O}^{0}(W P)>P_{H 2 O}^{0}(D W)$ because $T(W P)>T(D W)$, and therefore,

$R H(W P)<R H(D W)$, with $R H=P_{H 2 O} / P_{H 2 O}^{0}$,

where

$P_{H 2 O}$ is the partial pressure of water,

$P_{H 2 O}^{0}$ is the equilibrium vapor pressure of water,

and $W P$ and $D W$ indicate the waste package and drift wall, respectively.

Consequently, some of the water that contacts the DSs or WPOBs will necessarily evaporate in order for the remaining aqueous solutions to equilibrate with the local relative humidity. To understand the following sections, it is important first to understand the effect of concentrating the ionic species in the water, that is, which ions will remain in solution and which ions will form precipitates.

The deliquescence points define the lower $R H$ limit for which aqueous salt solutions will exist. The deliquescence points are the $R H$ s at which hygroscopic salts will sorb water from the atmosphere and form their most concentrated aqueous salt solutions. At higher values of $R H$, the aqueous solutions become more dilute. When these salt solutions are in contact with metals or alloys, electrochemical corrosion processes are enabled. Hence, it is the deliquescence point for a 


\section{CHECK COPY}

particular salt or salt mixture which defines the condition necessary for aqueous electrochemical corrosion at a particular temperature.

In Section 6.4, a discussion of the types of salt/mineral scale that can form on "heat transfer surfaces" is presented.

\subsection{THE EH AND PH OF AQUEOUS SOLUTIONS UNDER RELEVANT REPOSITORY CONDITIONS}

\subsubsection{Accessible Relative Humidity Conditions}

At the elevation of the potential repository the nominal atmospheric pressure is $87.67 \mathrm{kPa}$; this corresponds to a boiling point of $96^{\circ} \mathrm{C}$ for pure water. Figure $6-1$ shows the relative humidities that are accessible as a function of temperature for the fixed total pressure of $87.67 \mathrm{kPa}$. For comparison, the maximum relative humidity values are also plotted for a fixed total pressure of $101.32 \mathrm{kPa}$, with the corresponding boiling point of water equal to $100^{\circ} \mathrm{C}$.

The consequence of the fixed total system pressure is that the relative humidities decrease for temperatures above the boiling point of water, as shown in Figure 6-1.

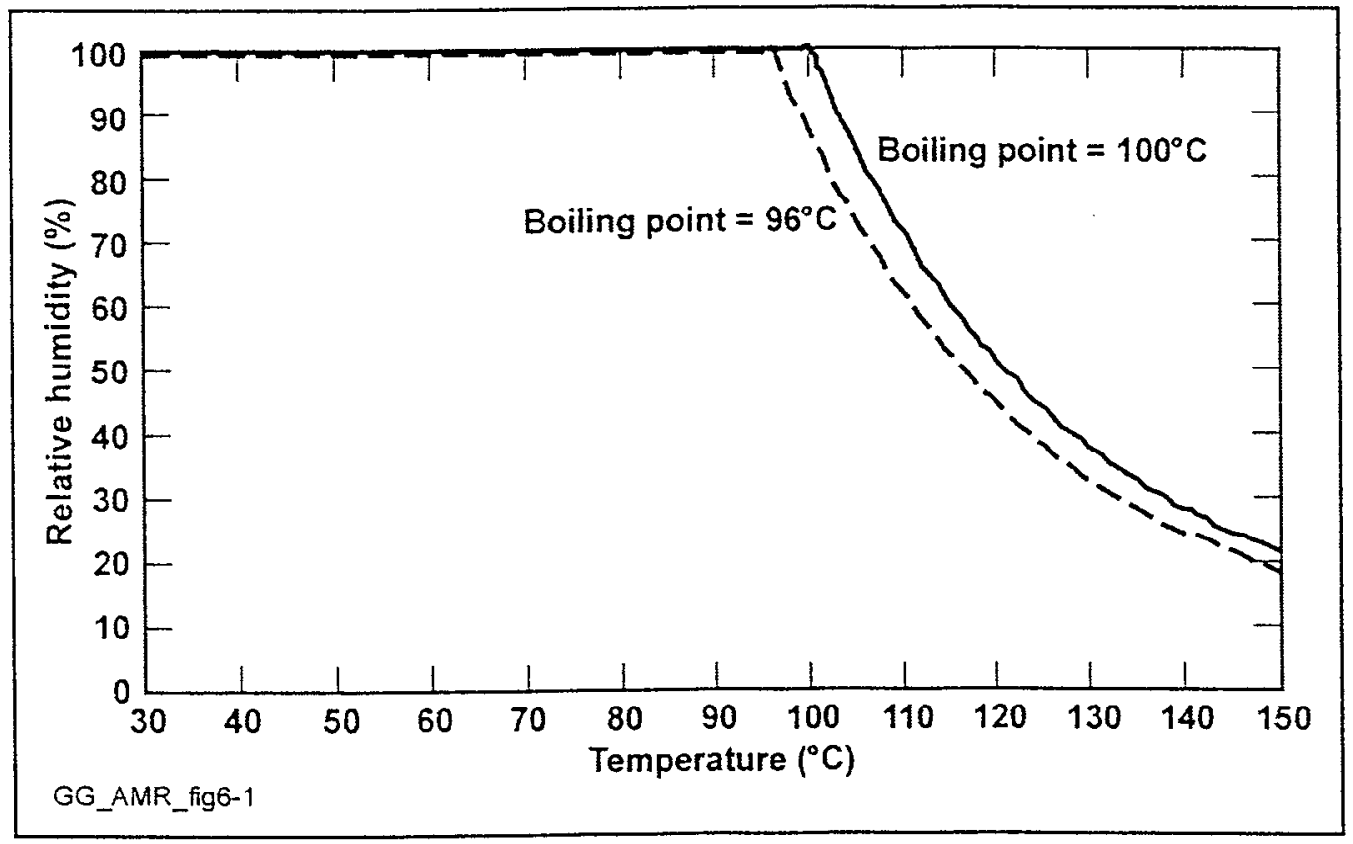

Figure 6-1. Maximum Relative Humidity versus Temperature for Boiling Points of $96^{\circ} \mathrm{C}$ and $100^{\circ} \mathrm{C}$

\subsubsection{Gas Phase Composition as a Function of Relative Humidity}

The following tables and accompanying figures show the variation of oxygen $\left(\mathrm{O}_{2}\right)$ and carbon dioxide $\left(\mathrm{CO}_{2}\right)$ content of the drift air as a function of temperature and relative humidity. Also included in the table is the water vapor pressure as a function of temperature (Weast and Astle 


\section{CHECK COPY}

1981). Water $\left(P_{\mathrm{H} 2 \mathrm{O}}\right)$, carbon dioxide $\left(P_{\mathrm{CO} 2}\right)$ and oxygen $\left(P_{\mathrm{O}_{2}}\right)$ partial pressure were calculated according to the following:

$$
\begin{aligned}
& P_{H 2 O}=x_{R H} \times P_{H 2 O}^{0}, \\
& P_{C O 2}=x_{C O 2} \times\left(P_{t o t a l}-x_{R H} \times P_{H 2 O}^{0}\right), \text { and } \\
& P_{O 2}=x_{O 2} \times\left(P_{\text {total }}-x_{R H} \times P_{H 2 O}^{0}\right)
\end{aligned}
$$

where

$\begin{array}{ll}x_{\mathrm{CO} 2} & \text { carbon dioxide volume fraction of air } \\ x_{\mathrm{O} 2} & \text { oxygen volume fraction of air } \\ P_{u t a l} & \text { total atmospheric pressure } \\ x_{R H} & \text { fractional relative humidity } \\ P_{H 2 O}^{0} & \text { water vapor pressure. }\end{array}$

Figures 6-2 and 6-3 are plots of the calculations for the oxygen and carbon dioxide partial pressures for two scenarios. One case assumes that the partial pressure of water is always equal to its vapor pressure. The other scenario assumes that $R H=90 \%$ for $T \leq 100^{\circ} \mathrm{C}$ and $P_{H Z O}=0.90 \times P_{\text {total }}$ for $T>100^{\circ} \mathrm{C}$.

\subsubsection{Oxygen Solubility}

The electrochemical potential of an aqueous solution is a determinant of the corrosion processes that can occur on a metal surface. Dissolved oxygen strongly influences the electrochemical potential of the aqueous solution. Oxygen solubility in pure water and various salt solutions (in particular, $\mathrm{NaCl}$ ) have been studied by Cramer (1980).

The mole fraction, $x_{O 2}$ of molecular oxygen in the aqueous solution with some simplifying assumption vas given by:

$x_{O 2}=P_{O 2} / k$

where $P_{O_{2}}$ is the partial pressure of oxygen in the gas phase and $k$ is the Henry's Law constant. An analytical expression for $k$ was given as follows:

$\ln k=a_{0}+a_{1} / T+a_{2} / T^{2}+a_{3} / T^{3}+a_{4} / T^{4}$

where $k$ is in $\mathrm{MPa}$ and $\mathrm{T}$ in $\mathrm{K}$. The constants $a_{0}, a_{1}, a_{2}, a_{3}$, and $a_{4}$ are given in Table 4-14. 


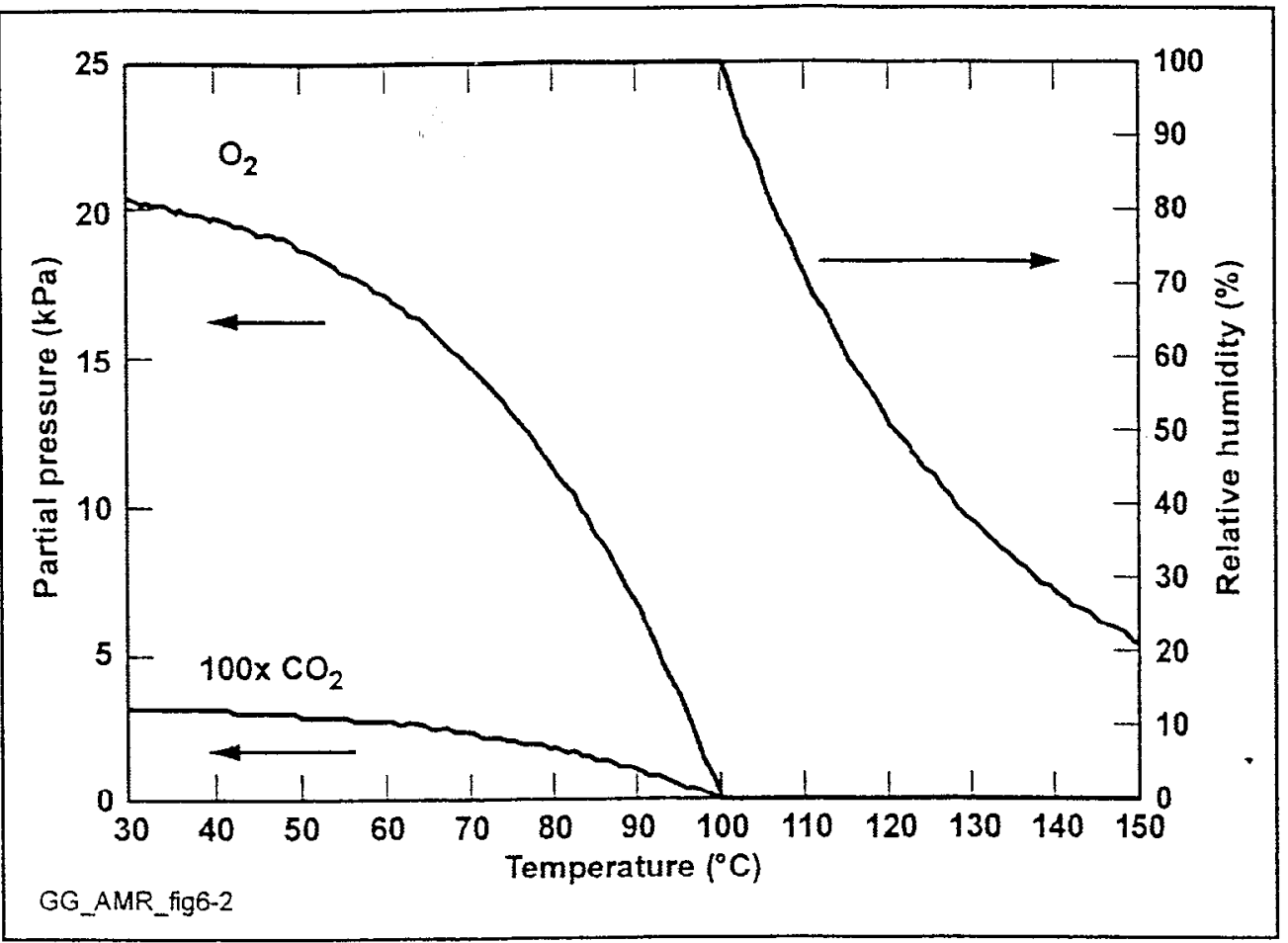

Figure 6-2. Calculated Partial Pressure of $\mathrm{O}_{2}$ and $\mathrm{CO}_{2}$ Assuming $\mathrm{H}_{2} \mathrm{O}$ Partial Pressure is at its Maximum; That is, $100 \% \mathrm{RH}$ for $\mathrm{T} \leq 100^{\circ} \mathrm{C}$ and $101.32 \mathrm{kPa}$ for $\mathrm{T}>100^{\circ} \mathrm{C}$

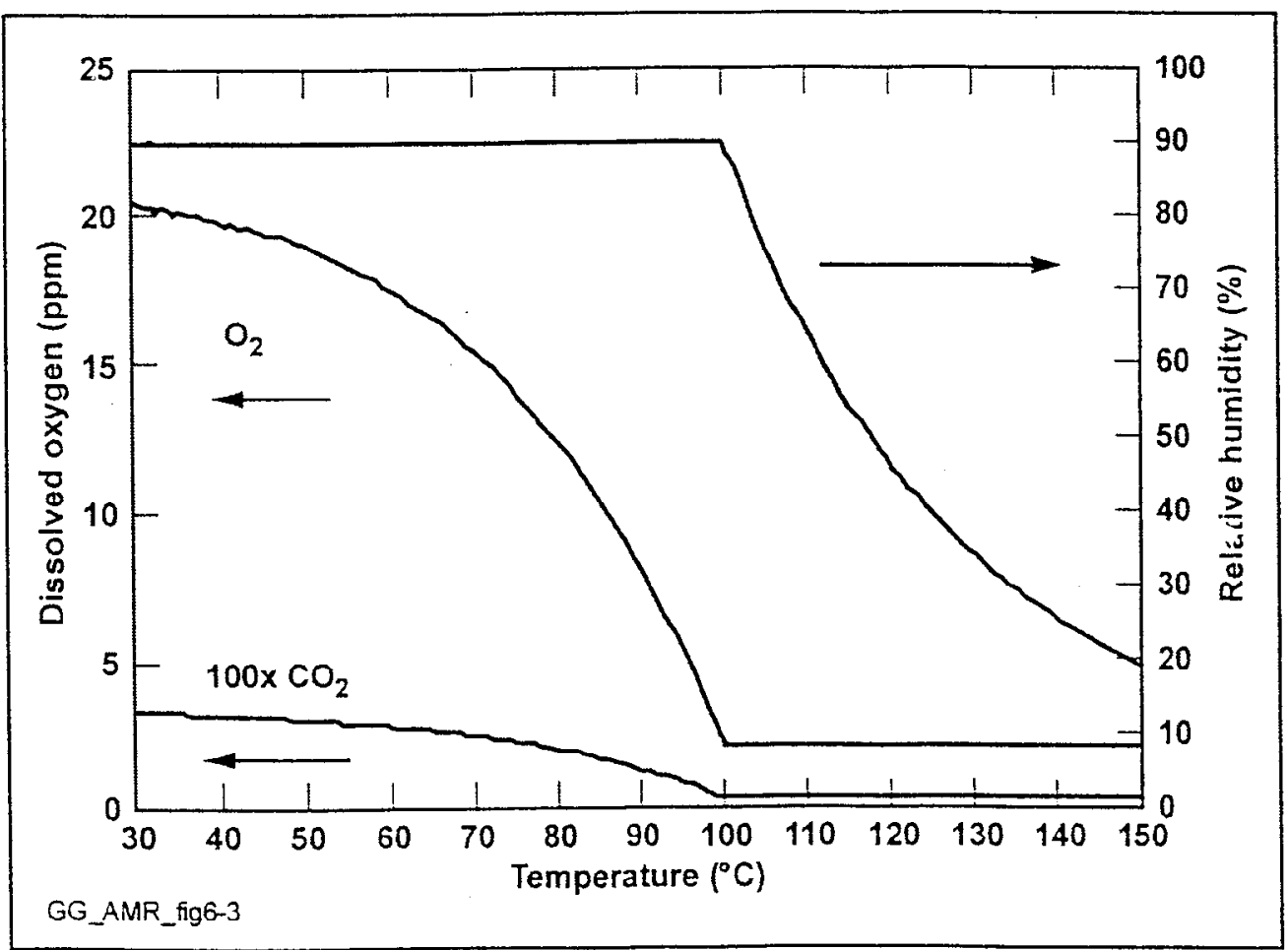

Figure 6-3. Calculations of $\mathrm{O}_{2}$ and $\mathrm{CO}_{2}$ Partial Pressures Assuming $\mathrm{RH}=90 \%$ for $T<100^{\circ} \mathrm{C}$ and the Partial Pressure of Water is Equal to 0.9 Times the Total System Pressure Above $100^{\circ} \mathrm{C}$ 


\section{CHECK COPY}

The solubility of oxygen in parts per million (ppm) by weight is given by:

$D_{O 2}(p p m)=\left(P_{O 2} / k\right) \times\left(1000 / M W_{H 2 O}+M_{\text {solute }}\right) \times M W_{O 2} / \rho_{\text {so In }}$

where

1000 weight one liter of the solvent in grams

$M W_{H 2 O} \quad$ molecular weight of water $(18.0153 \mathrm{gm} / \mathrm{gm}-\mathrm{mol})$

$M W_{O 2} \quad$ molecular weight of $\mathrm{O}_{2}$

$M_{\text {solute }} \quad$ molarity of the solute and

$\rho_{\text {soln }} \quad$ density of the solution in gm/liter.

The density of the fluid is given by

$\rho_{s o \text { In }}=\rho_{H 2 O}+\left(0.03378+0.5622 \times 10^{-5} \times \exp (T / 66.0)\right) \times \rho_{H 2 O} \times m_{s o \text { In }}$

where

$\rho_{H 2 O} \quad$ density of water

$m_{\text {soln }} \quad$ molality of solutions.

Plotted in Figures 6-4 and 6-5 are the solubilities of oxygen in three aqueous solutions for two cases of different partial pressures of oxygen with a fixed total pressure. In Figure 6-4, the partial pressure of $\mathrm{H}_{2} \mathrm{O}$ is assumed to be at its maximum value, i.e., $100 \%$ RH below $100^{\circ} \mathrm{C}$ and 101.32 $\mathrm{kPa}$ above $100^{\circ} \mathrm{C}$. Above $100^{\circ} \mathrm{C}$ there is no partial pressure of oxygen-therefore no oxygen is in any of the solutions. Below $100^{\circ} \mathrm{C}$ the partial pressure of oxygen is steadily increasing with decreasing temperature (see Figure 6-2) and hence the oxygen solution concentrations are increasing. Fvident in Figure 6-2 is the "salting out" effect; that is, the solutes in solution cause a decrease in the oxygen solubility for a given partial pressure of oxygen. It worth noting that the salt solutions are not in equilibrium with the water vapor pressure under these conditions. Under the condition of $100 \% \mathrm{RH}$, waters in equilisrium with the surrounding gas phase are infinitely dilute. Therefore, both aqueous salt solutions would tend to become more dilute over time. The equilibrium concentration dependence on $\mathrm{RH}$ is discussed in Section 6.3.1. 


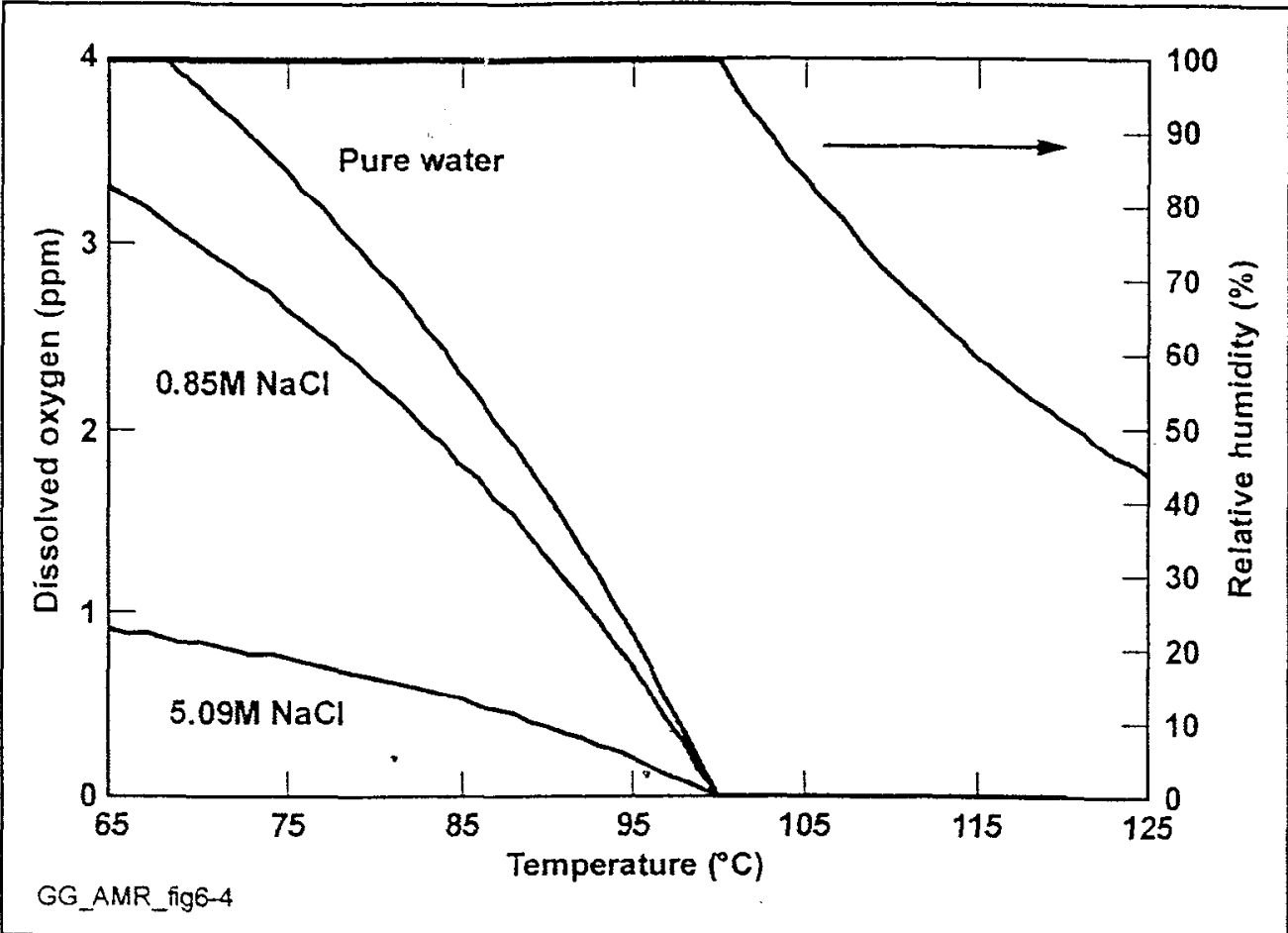

Figure 6-4. Dissolved Oxygen Concentration (ppm) for Three Aqueous Solutions. Water Partial Pressure: $100 \% \mathrm{RH}$ for $\mathrm{T} \leq 100^{\circ} \mathrm{C}$ and $101.32 \mathrm{kPa}$ for $\mathrm{T}>100^{\circ} \mathrm{C}$

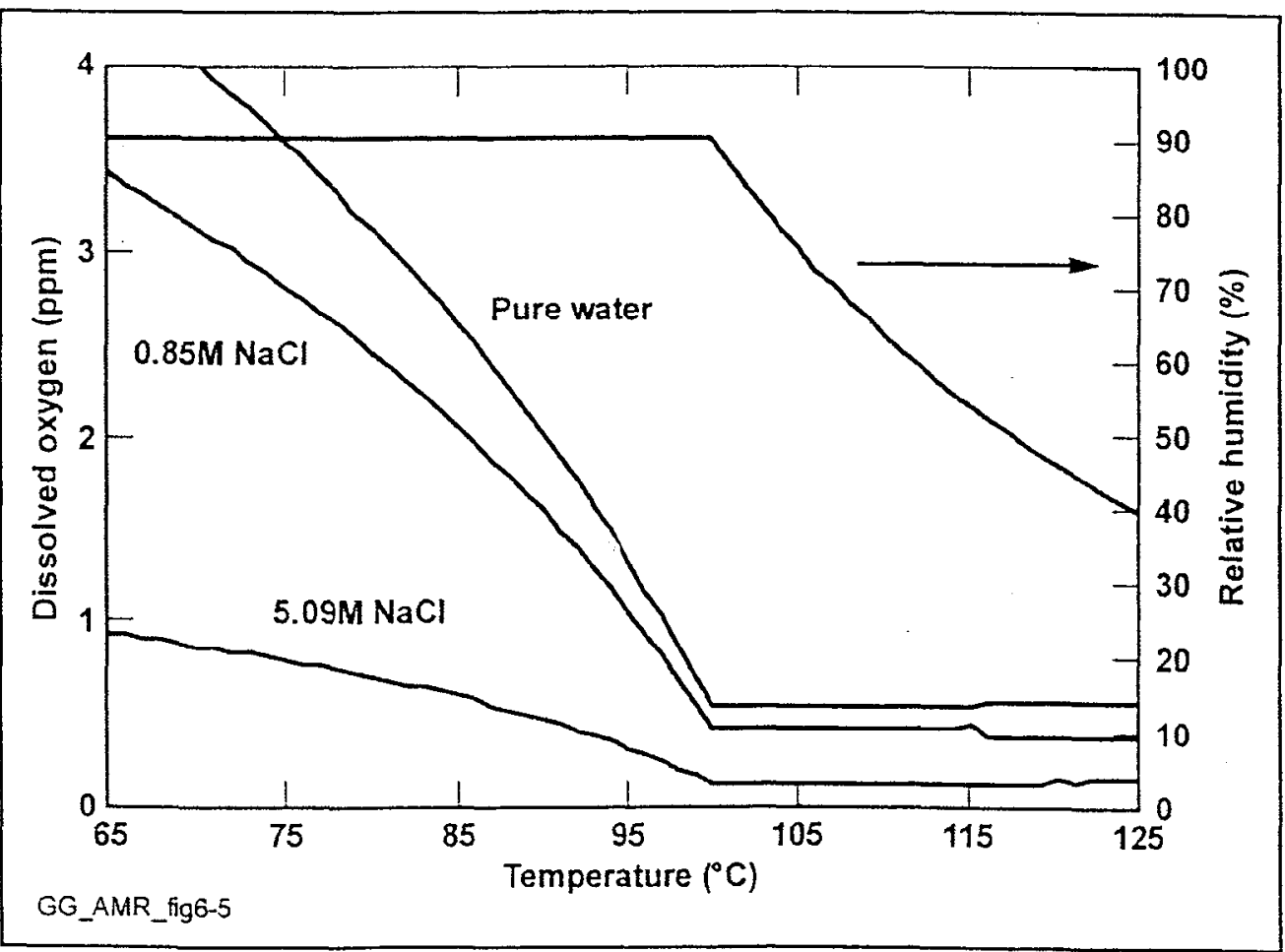

Figure 6-5. Dissolved Oxygen Concentration (ppm) for Three Aqueous Solutions. Partial Pressure of Water Determined by: $90 \% \mathrm{RH}$ for $\mathrm{T} \leq 100^{\circ} \mathrm{C}$ and 87.67 for $\mathrm{T}>100^{\circ} \mathrm{C}$ 


\section{CHECK COPY}

In Figure 6-5 the dissolved oxygen content is calculated for the three aqueous solutions assuming that the water partial pressure is determined by the following conditions: $90 \% \mathrm{RH}$ for $\mathrm{T} \leq 100^{\circ} \mathrm{C}$ and $87.67 \mathrm{kPa}$ for $\mathrm{T}>100^{\circ} \mathrm{C}$. For all the solutions, a significant dissolved oxygen concentration is evident above $100^{\circ} \mathrm{C}$. As in the previous example, for at least a portion of the $T$ and $\mathrm{RH}$ the aqueous solutions are not in thermodynamic equilibrium with the environmental conditions. Bulk pure water is not in equilibrium with the vapor conditions at any temperature, and would evaporate. The $5.09 \mathrm{M} \mathrm{NaCl}$ solution would be in equilibrium with the vapor only when the relative humidity is approximately $75 \%$. At lower $\mathrm{RH}$ values it would tend to concentrate, and at still lower it would form a dry salt. At higher RH values, it would tend to form more dilute solutions. The $0.85 \mathrm{M} \mathrm{NaCl}$ solution is approximately in equilibrium with the $90 \% \mathrm{RH}$ conditions. A solution, which is in equilibrium with the specified vapor, would form an aqueous solution at $109^{\circ} \mathrm{C}$ with some dissolved oxygen. With decreasing temperature (and hence, increasing $\mathrm{RH}$ ) the solution will become more dilute, with a consequent rise in the dissolved oxygen content. At temperatures below $100^{\circ} \mathrm{C}$ the oxygen solubility curve would approximately follow the curve for the $0.85 \mathrm{M} \mathrm{NaCl}$ solution.

\subsubsection{Determination of the Electrochemical Potential of Aqueous Solutions as a Function of Oxygen Partial Pressure or Equivalently Oxygen Solubility (TBD)}

The electrochemical potential $(\mathrm{eH})$ is a very strong determinant of the electrochemical corrosion processes that a metal alloy can be subjected to. Testing will be performed to determine the eH of relevant aqueous salt solutions under various relevant oxygen partial pressures and temperatures.

\subsection{5 $\mathrm{pH}$ Control by the $\mathrm{CO}_{2}-\mathrm{HCO}_{3}-\mathrm{CO}_{3}{ }^{2-}$ System (Stumm and Morgan 1981)}

The $\mathrm{pH}$ of well J13-type waters is strongly influenced by the $\mathrm{CO}_{2(a q)}-\mathrm{HCO}_{3}^{-}-\mathrm{CO}_{3}^{2-}$ system (bicarbonate/carbonate). The following chemical equations defined this system:

$$
\begin{aligned}
& \mathrm{CO}_{2(g)} \Leftrightarrow \mathrm{CO}_{2(a q)} \\
& \mathrm{CO}_{2(a q)}+\mathrm{H}_{2} \mathrm{O} \Leftrightarrow \mathrm{H}^{+}+\mathrm{HCO}_{3}^{-} \\
& \mathrm{HCO}_{3}^{-}+\mathrm{OH}^{-} \Leftrightarrow \mathrm{H}_{2} \mathrm{O}+\mathrm{CO}_{3}^{2-} \\
& \mathrm{H}_{2} \mathrm{O} \Leftrightarrow \mathrm{H}^{+}+\mathrm{OH}^{-}
\end{aligned}
$$

In balance requires that

$$
\left[\mathrm{H}^{+}\right]=\left[\mathrm{HCO}_{3}^{-}\right]+2\left[\mathrm{CO}_{3}^{2-}\right]+\left[\mathrm{OH}^{-}\right]
$$

and the equilibrium constant for water dissociation $\left[\mathrm{H}_{2} \mathrm{O}\right] \Leftrightarrow\left[\mathrm{OH}^{\prime \prime}\right]\left[\mathrm{H}^{+}\right]$is given by

$$
K_{W}=\left[O H^{-}\right]\left[H^{+}\right]
$$




\section{CHECK COPY}

Under some circumstances the partial pressure of gaseous carbon dioxide $\left(\mathrm{CO}_{2(g)}\right)$ will be very low because of the large partial pressure of water excluding air, which causes a loss of aqueous carbon dioxide, $\mathrm{CO}_{2(u q)}$. The aqueous carbon dioxide will be replenished by recombination of the bicarbonate ion with the hydronium ion (eq. 2). By considering the ion balance and water dissociation equations, it can be shown that the $\mathrm{pH}$ of the solution increases as the partial pressure of carbon dioxide decreases.

\subsubsection{Quantify the Dependence of the Aqueous Solution pH on the Partial Pressure of Carbon Dioxide as a Function of Temperature and Water Partial Pressure (TBD)}

Experimental work and computer simulations will be performed to characterize the $\mathrm{pH}$ dependence of the aqueous solutions, especially under conditions of elevated temperatures and low carbon dioxide partial pressures. This work will be done to determine if very high $\mathrm{pH}$ solutions could form under expected repository conditions. Very high $\mathrm{pH}$ solutions may change the operative corrosion processes.

\subsection{WATER ADSORPTION ON SURFACES IN THE ABSENCE OF HYGROSCOPIC SALTS}

\subsubsection{Water Adsorption on Flat Surfaces as a Function of Temperature and Relative Humidity}

In the absence of hygroscopic salts, only limited amounts of water will adsorb on transition metal or metal oxide surfaces. The data of Lee and Staehle (1997) show that the amount of water adsorbed is somewhat independent of temperature, but is a function of relative humidity. (See Figures 4-1 and 4-2.) Estimates of water film thickness have been made from this data by assuming microscopically flat surfaces in their tests and a water density of $1 \mathrm{~g} / \mathrm{cm}^{3}$; for the purposes of this analysis the assumptions are reasonable. Film thickness layers are of the order of $10-100 \AA$.

It is important to note that bulk water condensation can occur only when $R H \geq 100 \%$, that is, where the atmosphere is supersaturated with water vapor.

The adsorbed water layer may facilitate the electrochemical reduction of oxygen when it is present. Assuming a fixed partial pressure of oxygen, steady state amounts of oxygen in the films are expected because of the very thin thickness of the films and the fast diffusion of the oxygen in water.

\subsubsection{Capillary Condensation as a Function of Relative Humidity and Temperature}

The condensation of water vapor in crevices in contact with the metal barrier surfaces is of concern when considering corrosion under humid air conditions (see Table 6-1). The Kelvin equation is used to estimate the conditions under which water vapor condensation occurs in crevices in contact with the surface or in defects in corrosion products on the surface. 
Table 6-1. Calculation of the Radius of a Pore in which Water Condensation will Occur. The Calculations were Performed using a Microsoft Excel Spreadsheet

\begin{tabular}{|c|c|c|c|c|c|c|}
\hline & & & \multicolumn{2}{|c|}{$\begin{array}{c}\text { Pore radius, } r, \\
(\mathrm{~A}) \text { or }\left(10^{-10} \mathrm{~m}\right)\end{array}$} & \\
\hline $\mathrm{RH}$ & $100^{\circ} \mathrm{C}$ & $80^{\circ} \mathrm{C}$ & $70^{\circ} \mathrm{C}$ & $60^{\circ} \mathrm{C}$ & $50^{\circ} \mathrm{C}$ & $40^{\circ} \mathrm{C}$ \\
\hline 0.99 & 710.2 & 786.5 & 827.6 & 871.2 & 917.1 & 965.3 \\
\hline 0.98 & 353.3 & 391.3 & 411.7 & 433.4 & 456.2 & 480.2 \\
\hline 0.97 & 234.3 & 259.5 & 273.1 & 287.5 & 302.6 & 318.5 \\
\hline 0.96 & 174.8 & 193.6 & 203.8 & 214.5 & 225.8 & 237.7 \\
\hline 0.95 & 139.2 & 154.1 & 162.2 & 170.7 & 179.7 & 189.1 \\
\hline 0.94 & 115.4 & 127.8 & 134.4 & 141.5 & 149.0 & 156.8 \\
\hline 0.93 & 98.4 & 108.9 & 114.6 & 120.7 & 127.0 & 133.7 \\
\hline 0.92 & 85.6 & 94.8 & 99.8 & 105.0 & 110.5 & 116.4 \\
\hline 0.91 & 75.7 & 83.8 & 88.2 & 92.8 & 97.7 & 102.9 \\
\hline 0.90 & 67.7 & 75.0 & 78.9 & 83.1 & 87.5 & 92.1 \\
\hline 0.85 & 43.9 & 48.6 & 51.2 & 53.9 & 56.7 & 59.7 \\
\hline 0.80 & 32.0 & 35.4 & 37.3 & 39.2 & 41.3 & 43.5 \\
\hline 0.75 & 24.8 & 27.5 & 28.9 & 30.4 & 32.0 & 33.7 \\
\hline 0.70 & 20.0 & 22.2 & 23.3 & 24.5 & 25.8 & 27.2 \\
\hline 0.65 & 16.6 & 18.3 & 19.3 & 20.3 & 21.4 & 22.5 \\
\hline 0.60 & 14.0 & 15.5 & 16.3 & 17.1 & 18.0 & 19.0 \\
\hline 0.55 & 11.9 & 13.2 & 13.9 & 14.6 & 15.4 & 16.2 \\
\hline 0.50 & 10.3 & 11.4 & 12.0 & 12.6 & 13.3 & 14.0 \\
\hline 0.45 & 8.9 & 9.9 & 10.4 & 11.0 & 11.5 & 12.1 \\
\hline 0.40 & 7.8 & 8.6 & 9.1 & 9.6 & 10.1 & 10.6 \\
\hline 0.35 & 6.8 & 7.5 & 7.9 & 8.3 & 8.8 & 9.2 \\
\hline 0.30 & 5.9 & 6.6 & 6.9 & 7.3 & 7.7 & 8.1 \\
\hline 0.25 & 5.1 & 5.7 & 6.0 & 6.3 & 6.6 & 7.0 \\
\hline 0.20 & 4.4 & 4.9 & 5.2 & 5.4 & 5.7 & 6.0 \\
\hline 0.15 & 3.8 & 4.2 & 4.4 & 4.6 & 4.9 & 5.1 \\
\hline 0.10 & 3.1 & 3.4 & 3.6 & 3.8 & 4.0 & 4.2 \\
\hline
\end{tabular}

The relative humidity at which condensation occurs in a pore of radius $r$ is given by Fyfe (1994):

$x_{R H}=\exp \left(P_{c} M W_{H_{2}} / \rho R T\right)$ with $P_{c}=2 \gamma / r$.

Rearranging the equation to define the radius as a function of temperature and relative humidity gives:

$r=-2 \gamma M W /\left(\rho R T \times \ln \left(x_{R H}\right)\right)$

where

$x_{R H} \quad$ fractional relative humidity

$M W_{H 2 O} \quad$ molecular weight $(\mathrm{kg} /$ mole $)$ 


\title{
CHECK COPY
}

\author{
$\rho \quad$ mass density of water $\left(\mathrm{kg} / \mathrm{m}^{3}\right)$ \\ $R \quad$ gas constant ( 8.31433 joules/K-mole) (Weast and Astle 1981) \\ $\gamma \quad$ surface tension of water-air (joules $/ \mathrm{m}^{2}$ ) \\ T temperature $(\mathrm{K})$.
}

Sample calculation for $T=353.15 \mathrm{~K}\left(80^{\circ} \mathrm{C}\right)$ and $x_{R H}=0.91$.

$r=-2 \cdot 0.0626$ joules $/ \mathrm{m}^{2} \cdot(18.0153 / 1000) / 0.97183 \mathrm{gm} / \mathrm{cm}^{3} \cdot(1 \mathrm{~kg} / 1000 \mathrm{gm}) \cdot\left(1000000 \mathrm{~cm}^{3} / \mathrm{m}^{3}\right) \cdot$ $(8.31433$ joules $/ \mathrm{K}$-mole $) \cdot 353.15 \mathrm{~K} \cdot \ln (0.91)$

$r=8.38 \times 10^{-9}$ meters.

\subsubsection{Relative Humidity Effect on the Oxidation (TBD) and Dissolution of the Candidate Materials}

The limited amount of water in the films due to adsorption under $\mathrm{RH}<100 \%$ is not sufficient to support a significant amount of dissolution of the candidate materials because of the very limited solubility of these material in near-neutral waters.

The oxidation of materials may be enhanced at high relative humidity because of the enhancement of the reduction of oxygen due to the adsorbed water film. Under these conditions the oxidation is expected to be controlled by the solid-state diffusion of ions through the oxide layer, just as it would be for dry oxidation. The adsorbed water film may contribute to the electrical potential across the oxide, which affects the ion diffusion. The extent of this enhancement will be investigated, but presently, it is not considered to be significant at the oxygen partial pressure expected.

\subsection{AQUEOUS SALT SOLUTIONS ON THE DSs AND WPOBs}

Aqueous solutions of hygroscopic salts on the DSs and WPOBs can occur as a result of several processes. The hygroscopic salts enable aqueous solutions to exist at relative humidities below $100 \%$. Hygroscopic salts may be deposited by aerosols and dust, and may be introduced with the backfill. They will be contained in the seepage water that enters the drifts and the episodic water that flows through the drifts.

If the salts are deposited under very low relative humidity conditions, then the aqueous solutions will form after the relative humidity exceeds the deliquescence point for the salt mixture present. At the deliquescence point, the solutions are very concentrated and may only consist of specific ions in the salt mixture. As the relative humidity increases the solutions will become more dilute and other ions in the salt mixture may become soluble.

If the salts contact the components as a result of seepage or episodic water flow at moderate to high relative humidity, then the aqueous solutions may result from the evaporative/boiling concentration of the solutions. The amount of concentration and precipitate formation depends 


\section{CHECK COPY}

on the relative humidity and temperature differences between the aqueous solution and the specific component.

It is possible that the temperature and relative humidity differences between the incoming water the engineered barrier system (EBS) component may be such that aqueous solutions will exist on the component under $\mathrm{T}$ and $\mathrm{RH}$ conditions that do not support those solutions. For example, ions may be in solution that would be expected to form precipitates. The extent and consequences of this are being investigated.

Sections 6.4.1-6.4.3 contain offer a basic understanding for the aqueous salt solutions that may form on the DSs and WPOBs, then discuss the experimental work that has been performed to date, and is ongoing.

\subsubsection{Deliquescence Points and Boiling Points for Pure Salts}

As stated in Section 6.1, it is the deliquescence point of a particular salt that defines the RH condition necessary for aqueous electrochemical corrosion processes of a metal with salt deposits to occur at a given temperature. Similarly, salt mixtures will have a characteristic deliquescence point that is dependent on the ions in the mixture.

The deliquescence points have been determined for a limited number of pure salts over a limited temperature range (Greenspan 1977). The data for various pure salts given in Table 4-8 are plotted in Figure 6-6. Included in the figure are plots for the maximum $R H$ obtainable for pure water with atmospheric conditions such that boiling points are $96^{\circ} \mathrm{C}\left(P_{\text {total }}=87.67 \mathrm{kPa}\right)$ and $100^{\circ} \mathrm{C}\left(P_{\text {toral }}=101.32 \mathrm{kPa}\right)$. Also included in the figure are the boiling points at sea level $\left(P_{\text {total }}=\right.$ $101.32 \mathrm{kPa}$ ) for some salts (Frazier 1928).

The boiling point for an aqueous salt solution is the temperature at which the equilibrium water vapor pressure of the salt solution is equal to the atmospheric pressure. The aqueous salt solution concentration is also at its maximum. Hence the $R H$ value at the boiling point is its deliquescence point.

For pure salts whose solubilities change little with temperature, the deliquescence points also change little with temperature. For instance, $\mathrm{NaCl}$, whose solubility changes from $35 \mathrm{gm} / 100$ $\mathrm{cm}^{3}$ of water@20 $20^{\circ} \mathrm{C}$ to $37 \mathrm{gm} / 100 \mathrm{~cm}^{3}$ of water $@ 100^{\circ} \mathrm{C}$ (see Table 4-8), the deliquescence points are nearly constant with temperature $(72 \%-75 \% \mathrm{RH})$. At the boiling point of $\mathrm{NaCl}$ the $\mathrm{RH}$ is $73.15 \%$. Similar behavior is seen for $\mathrm{KCl}$ and $\mathrm{K}_{2} \mathrm{SO}_{4}$.

In contrast, for pure salts whose solubility changes significantly with temperature, the deliquescence points also change significantly with temperature. For instance, $\mathrm{NaNO}_{3}$, whose solubility changes from $97 \mathrm{gm} / 100 \mathrm{~cm}^{3}$ of water @20 $\mathrm{C}$ to $197 \mathrm{gm} / 100 \mathrm{~cm}^{3}$ of water@100 $@$ (see Table 4-8), the deliquescence changes significantly with temperature from $85 \% @ 20^{\circ} \mathrm{C}$ to 70 @ $90^{\circ} \mathrm{C}$. At the sea-level boiling point $\left(120.6^{\circ} \mathrm{C}\right)$, the $\mathrm{RH}$ is $50.1 \%$. 


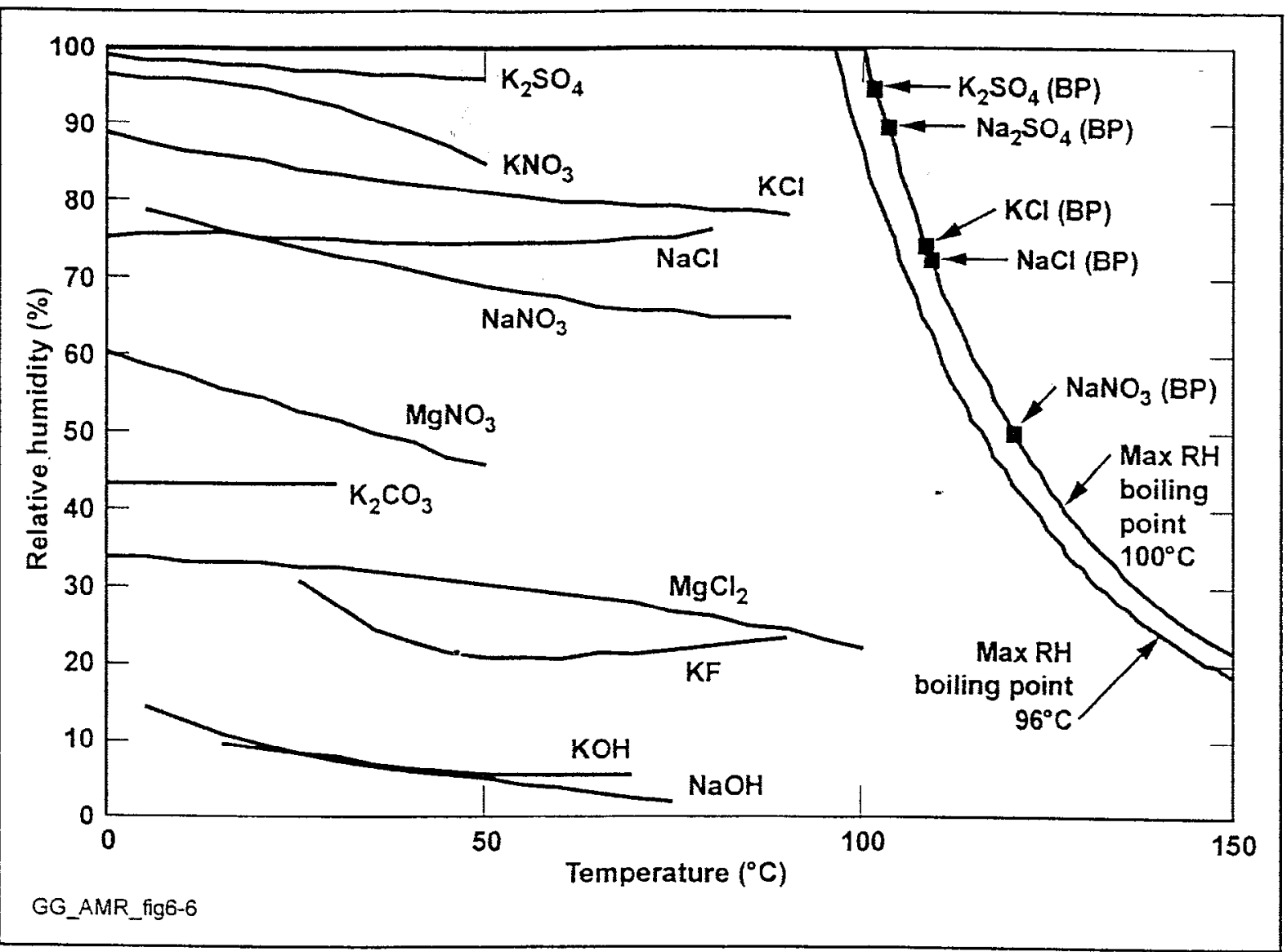

Figure 6-6. Deliquescence Points (Greenspan 1976) and Boiling Points for Several Pure Salts (Frazier 1928)

\subsubsection{RH Effect on Aqueous Solution Composition}

Decreasing the relative humidity of the atmosphere in contact with an aqueous solution of a pure salt will lead to the evaporative concentrating of the aqueous solution. Decreasing the RH below a certain value (the deliquescence point) will result in the precipitation of the salt. This process is illustrated in Figure 6-7.

For mixed salt solutions, decrewsing the relative humidity of the atmosphere will concentrate some of the ion in solution and cause the precipitation of some species. For instance, as discussed below, the initial concentrating of $J 13$ water causes the precipitation of calcium and magnesium species. 


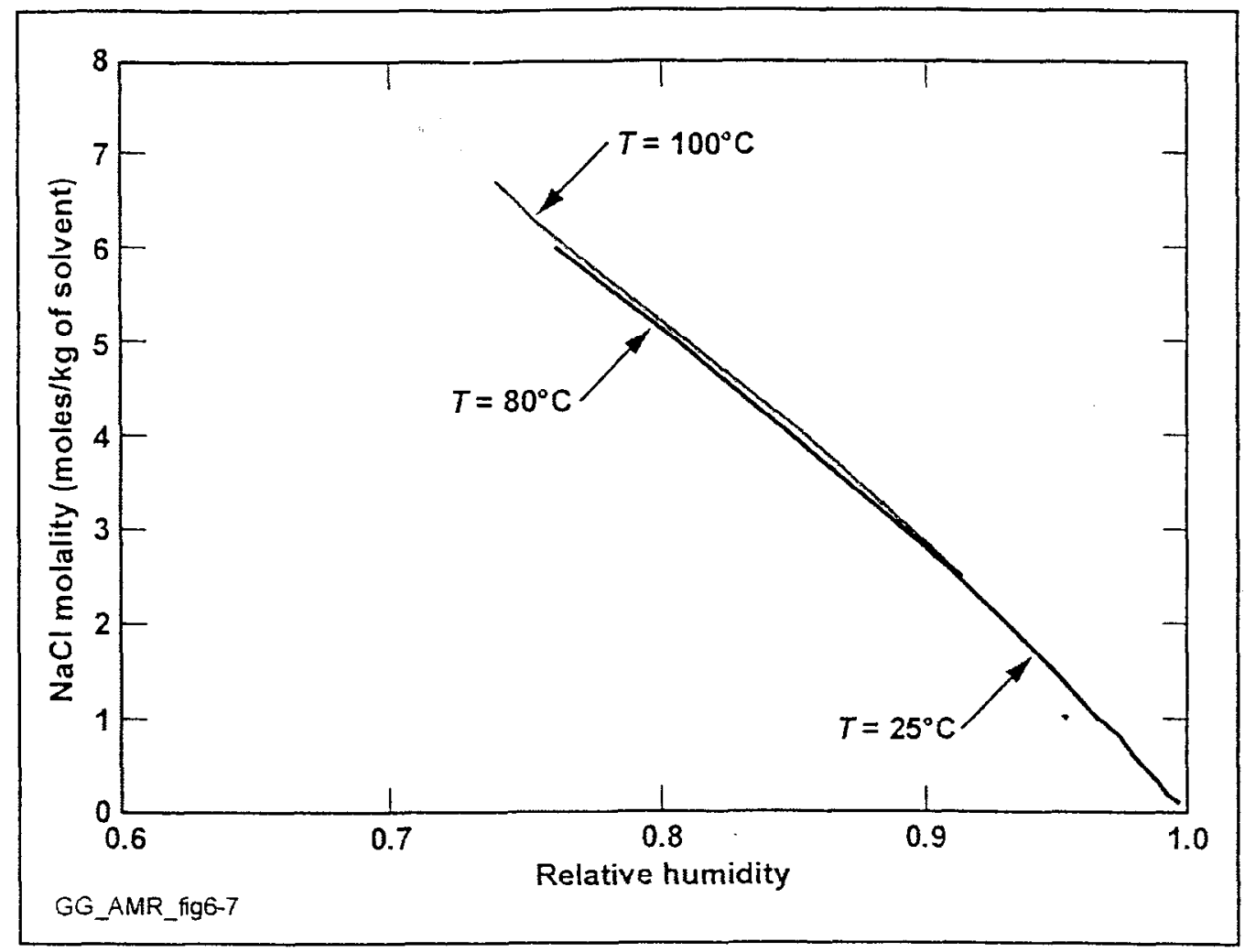

Figure 6-7. Aqueous NaCl Molality as a Function of Fractional Relative Humidity (Frazier 1928)

\subsubsection{Regions of Concern for the Aqueous Corrosion of DSs and WPOBs}

The DSs and WPOBs are expected to be most susceptible to electrochemical corrosion processes for temperatures above $80-85^{\circ} \mathrm{C}$. The temperature range of interest may extend to temperatures up to $120-125^{\circ} \mathrm{C}$; this uncertainty is due to the boiling point of $\mathrm{NaNO}_{3}$ solutions. The discussion above indicates that due to the temperature dependence of solute solubility and the concentration dependence on relative humidity, multiple compositions and concentrations as a function of temperature may be of interest for their effects on the corrosion susceptibility of DSs and WPOBs.

\subsection{BOILING/EVAPORATIVE CONCENTRATING OF SIMULATFD WELL J13 WATER}

The testing program has chosen to use well $\mathrm{J} 13$ water as a reference for waters that may contact the DSs and WPOBs. If a different composition of water is later deemed more appropriate, then an analysis will be performed to determine if that composition has a significant effect on the results presented in this section.

As discussed in Section 6.4, it is inevitable that the waters in contact with the DSs and the WPOBs will be more concentrated than the ambient waters at Yucca Mountain or the seepage water that enters the drifts. Several tests are ongoing to characterize the changes that occur upon boiling/evaporative concentrating of simulated well $J 13$ and simulated slightly concentrated well 


\section{CHECK COPY}

J13 water. These tests include the evaporative concentrating of simulated well J13 water that is dripped onto heated crushed tuff that is at or near the boiling point of the concentrated solution, and evaporative concentrating of simulated well $\mathrm{J} 13$ water that is dripped onto crushed tuff under conditions of constant temperature and relative humidity.

\subsubsection{Initial Concentrating of Well J13 Water}

It is expected that the initial concentrating of well $\mathrm{J} 13$ water will lead to the precipitation of Caand $\mathrm{Mg}$-based salts/minerals because of the very low solubility of the $\mathrm{Ca}$ and $\mathrm{Mg}$ in high bicarbonate/carbonate waters in neutral/alkaline aqueous solutions (See Section 4.1.8). The composition of well J13 water is given in Table 4-13. The largest anionic component of the water is the carbonate/bicarbonate couple (total bicarbonate concentration of $148 \mathrm{ppm}$ ). Calcium and magnesium concentrations are 13 and 2 ppm respectively. Evaporative concentrating of the aqueous solutions will exceed the solubility limits for the calcium and magnesium carbonates. The removal of $\mathrm{Mg}$ and $\mathrm{Ca}$ ions from solution by concentrating simulated well $\mathrm{J} 13$ waters has been documented experimentally (see Section 6.5.2). The magnesium seems to be removed to a greater extent than the calcium; however both are removed to a much greater extent than the $\mathrm{Na}$ and $\mathrm{K}$ ions. In the discussions that follow particular emphasis is placed on the water chemistry of sodium $(\mathrm{Na})$ and potassium $(\mathrm{K})$ salts because these are expected to be the dominant cation species.

\subsubsection{Evaporative Concentrating of a Simulated Well J13 by Boiling}

The results of this testing are presented in Section 4.1.17. The following figures are plots of a concentration factor defined as the ratio of the concentration in the solution to the concentration in the initial solution. The concentration factor for the cations is shown in Figure 6-8. As expected, the $\mathrm{Na}^{+}$and $\mathrm{K}^{+}$ion concentrations are continuously increasing in the solution. Also as expected, the $\mathrm{Mg}^{2+}$ and $\mathrm{Ca}^{2+}$ ion concentrations are very low. The one high value for $\mathrm{Ca}^{2+}$ that occurred in the first sampling does not persist at the later samplings.

The concentration factors for the anions show some interesting trends with continued concentration (see Figure 6-9). The $\mathrm{Cl}^{-}$and $\mathrm{NO}_{3}{ }^{-}$concentrations are continuously increasing with sampling. The $\mathrm{F}^{-}$concentration appears to be approaching very small values with increasing concentration. The $\mathrm{SO}_{4}{ }^{-}$concentration, while initially increasing with the $\mathrm{Cl}^{-}$and $\mathrm{NO}_{3}{ }^{-}$ concentrations, appears to be remaining constant or decreasing with concentrating.

\subsubsection{Determination of the $\mathrm{pH}$ of the Highly Concentrated Elevated Temperature Solutions (TBD)}

The in situ determination of the $\mathrm{pH}$ of highly concentrated solutions at elevated temperatures is required to fully characterize the solutions. Specialized electrodes are required. 


\section{CHECK COPY}

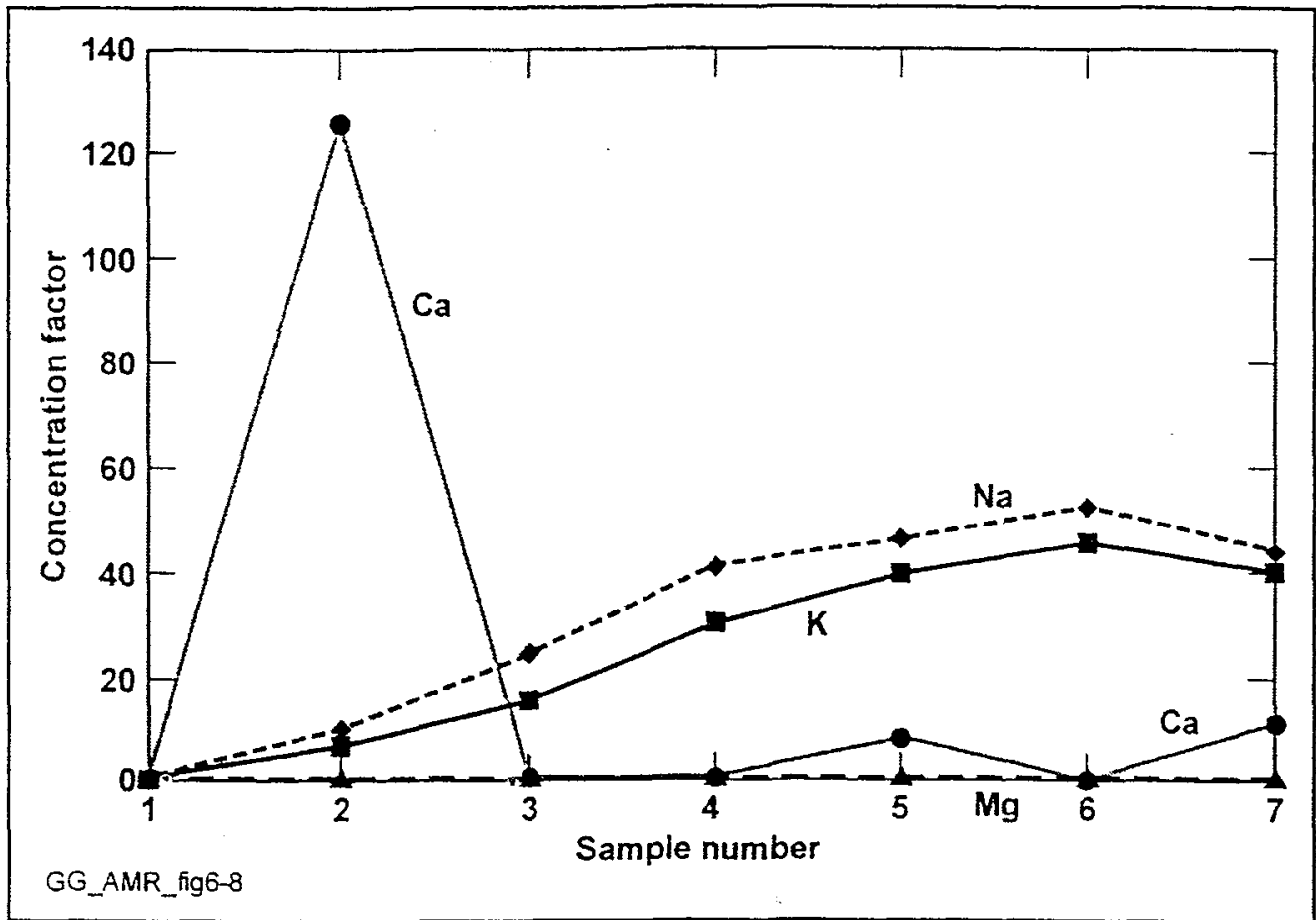

Figure 6-8. Concentration Factors for the Cations for the Evaporative Concentration of Simulated Well J13 Water Near the Boiling Point.

The First Sample Corresponds to the Initial Solution. Data in Section 4.1.17

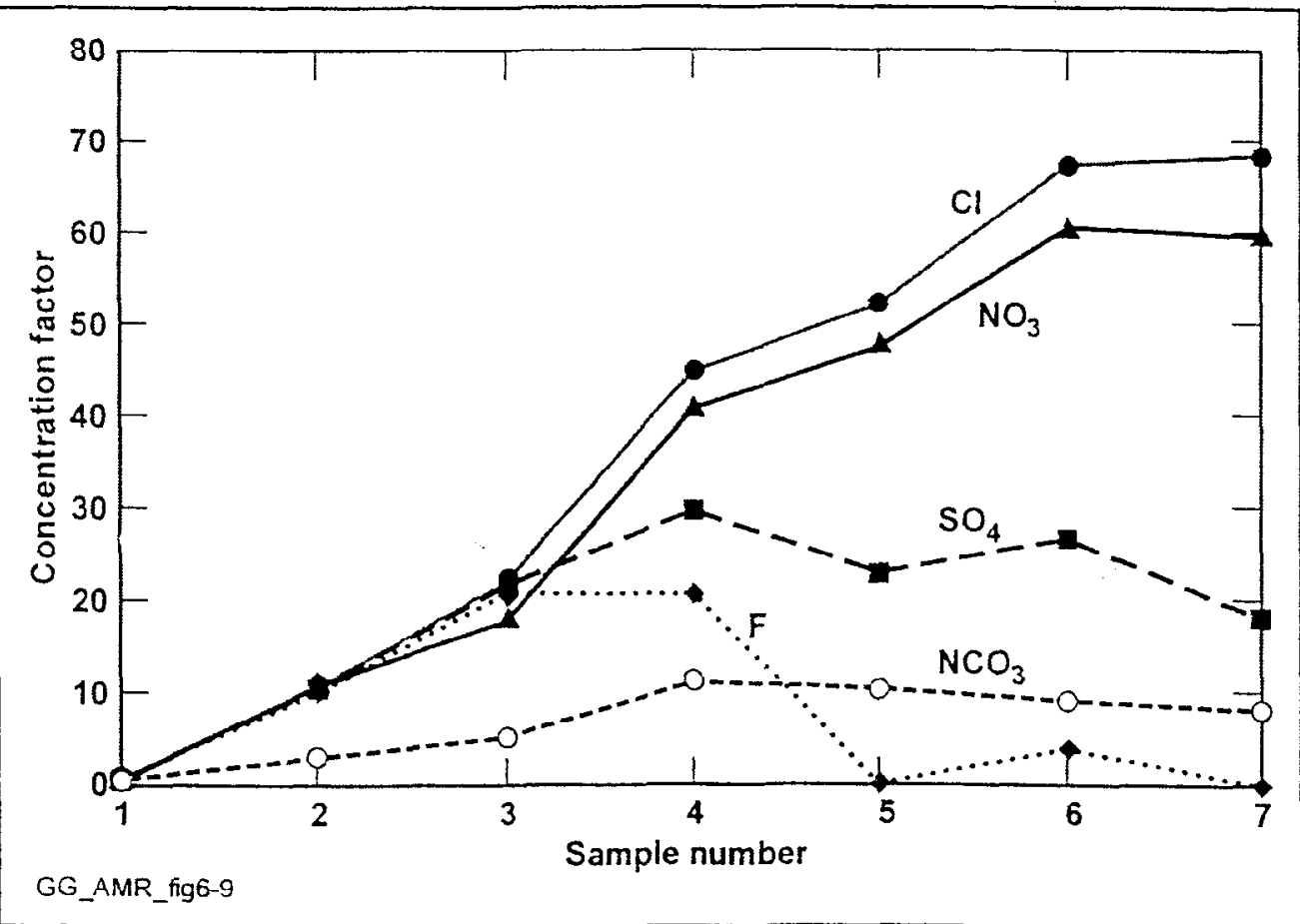

Figure 6-9. Concentration Factors for the Anions for the Evaporative Concentration of Simulated Well J13 Water Near the Boiling Point.

The First Sample Corresponds to the Initial Solution. Data in Section 4.1.17 


\section{CHECK COPY}

\subsubsection{Water Composition as a Function of Relative Humidity at Fixed Temperatures (DTN \#LL990703005924.083 and TBD\#)}

The objective of this work is to characterize the waters that would result from the evaporative concentration of waters contacting the WPOBs and the DSs in the range of temperature/relative humidity where candidate materials might be susceptible to corrosion. Testing has begun at $90^{\circ} \mathrm{C}$ and will continue at regular temperature intervals. The results are reported for evaporation at $85 \%$ RH. The starting solution was simulated 100 -times concentrated well J13 water. The aqueous solution dripped through a column of crushed tuff and collected in a beaker at the outlet of the column. Additional evaporation of the aqueous solution occurred in the beaker.

In Table 6-2 a concentration factor, which is the ratio of a specific ion concentration of the sample solution to the specific ion concentration of the initial solution, is presented. The data show that under these conditions calcium, magnesium, bicarbonate (as an indicator of total carbonate) and possibly fluoride are not concentrated to the extent that chloride, nitrate, sulfate, sodium, and potassium are. This result is consistent with the expected precipitation of carbonate species, and with the initial precipitation of some fluoride-containing salts/minerals.

Table 6-2. Results of the Evaporative Concentration of Simulated Well J13 Water Under Conditions of Constant Temperature and Relative Humidity

\begin{tabular}{|l|c|c|c|}
\hline Species & $\begin{array}{c}\text { Starting } \\
\text { Sol'n } \\
\text { (mg/liter) }\end{array}$ & $\begin{array}{c}\text { Final Sol'n } \\
85 \% R H / 90^{\circ} \mathrm{C} \\
(\mathrm{mg} / \text { /lter })\end{array}$ & $\begin{array}{c}\text { Concentration } \\
\text { Factor }\end{array}$ \\
\hline Sample \# & $\begin{array}{c}\text { Initial J13 } \\
\text { Batch A }\end{array}$ & J13-01 & \\
\hline $\mathrm{DTN}$ \#LL990703005924.083 & & \\
\hline $\mathrm{Cl}$ & & 15200 & 21.8 \\
\hline $\mathrm{NO}_{3}$ & & 14600 & 20.7 \\
\hline $\mathrm{SO}_{4}$ & & 30400 & 19.3 \\
\hline $\mathrm{F}$ & & 3500 & 18.1 \\
\hline $\mathrm{HCO}{ }^{*}$ & & 11370 & 14.4 \\
\hline $\mathrm{Na}$ & & 79700 & 20.2 \\
\hline $\mathrm{K}$ & & 10020 & 19.4 \\
\hline $\mathrm{Ca}$ & & 26 & 4.5 \\
\hline $\mathrm{Mg}$ & & 0 & 0 \\
\hline
\end{tabular}

*Total inorganic carbon expressed as bicarbonate.

\subsubsection{Salts/Minerals Formed Due to Evaporation of a Simulated J13 Water} (TBD)

Identification of salt/mineral phases, which result from the evaporative drying of simulated J13 water, will be performed on the precipitates that formed during the evaporative/boiling concentration testing. This will give an indication of the scale-forming minerals. They will also give an indication of the solutions that may form after the relative humidity increases above the deliquescence point of the salt mixtures. 


\section{CHECK COPY}

\subsection{THE EXISTENCE OF AQUEOUS SOLUTIONS ON THE EBS COMPONENTS UNDER ENVIRONMENT CONDITIONS THAT DO NOT SUPPORT THOSE SOLUTIONS (TBD)}

Aqueous solutions may exist on the EBS components where the evaporation rate is slower than the seepage flow of additional water. Under these conditions aqueous solutions may exist at higher temperatures or lower relative humidities than would normally support those solutions. The extent to which this may occur is being investigated to understand if there would be an increase in the aggressiveness of the aqueous solutions contacting the EBS components.

\subsection{ESTIMATE OF THE COMPOSITION OF CORROSIVELY AGGRESSIVE AQUEOUS SOLUTION THAT COULD RESULT FROM CONCENTRATING YUCCA MOUNTAIN WATERS}

To estimate the upper bound for the boiling point of waters that might contact the DSs and WPOBs surfaces the following scenario is postulated. In the near field, refluxing of water along the fractures will result in the chromatographic separation of the salts by their hygroscopic and solubility characteristics. That is, the salts will separate with the most soluble and hygroscopic salts migrating to the regions of lowest relative humidity, while the least soluble and hygroscopic will remain in the regions of highest relative humidity.

In terms of the salts present in the waters at Yucca Mountain, it is expected that the chloride and nitrate salts of sodium and potassium are the most likely to be the most soluble and hygroscopic salts remaining in solution upon concentrating Yucca Mountain type water. During the initial concentrating of the Yucca Mountain water, the calcium and magnesium ions are expected to form rather insoluble carbonate and silicate precipitates. With the calcium and magnesium ions removed from solution, it is the chloride, fluoride, sulfate, and nitrate salts of sodium and potassium that will remain in solution. As shown in Table 4-9, NaF has very limited solubility, and the sulfate salts of $\mathrm{Na}$ and $\mathrm{K}$ have also have limited solubilities.

It is expected that further concentration of the aqueous solution would ultimately result in nitratebased solution with small amounts of other ions, most notably the chloride ions. In order for the solution to remain aggressive toward corrosion, it was decided that the solution, in addition to being a high-temperature boiling solution, must also contain an aggressive ion, such as chloride. It was decided that the aggressive high boiling solution would be a mixture of chloride and nitrate salts of potassium and sodium.

At $100^{\circ} \mathrm{C}$, the solution was made by formulating a saturated solution of $\mathrm{NaNO}_{3}$, and then adding an additional amount of $\mathrm{KCl}$. The amount of $\mathrm{KCl}$ added corresponded to a $\mathrm{Cl}$ ion concentration of $3.6 \mathrm{M}$. Similarly, at $120^{\circ} \mathrm{C}$, the solution was made by increasing the $\mathrm{NaCO}_{3}$ nearly to its saturation limit, and then adding the amount of $\mathrm{KCl}$ that produces a $\mathrm{Cl}$ ion concentration of $4.3 \mathrm{M}$.

\subsection{CHARACTERIZATION OF MINERAL/SALT SCALE FORMATION ON METAL SURF ACES (TBD)}

The predominant scale-formers in Yucca Mountain type waters are calcium carbonate and silica (Cowan and Weintritt 1976). The tendency for carbonate scale formation is greatly enhanced by 
the concentration of the ions by water evaporation, provided the aqueous solution remains alkaline. Silica deposition will also be enhanced by water evaporation, provided that the solution's pH does not reach alkaline values where the silica solubility is greatly enhanced.

Scale can protect the underlying material if it forms a dense adherent layer. However, scale can have a deleterious effect on the underlying material if it forms a porous layer or a crevice with the underlying material.

The objective of this study is to characterize the scale that forms on the candidate materials for the DS and the WPOB under conditions simulating the repository environment. 


\section{CHECK COPY}

\section{CONCLUSIONS}

The major cations in Yucca Mountain waters are sodium $(\mathrm{Na})$, potassium $(\mathrm{K})$, calcium $(\mathrm{Ca})$, magnesium $(\mathrm{Mg})$, and silicon (Si). The major anions in Yucca Mountain water are chloride $(\mathrm{Cl})$, fluoride $(\mathrm{F})$, nitrate $\left(\mathrm{NO}_{3}\right)$, sulfate $\left(\mathrm{SO}_{4}\right)$, and bicarbonate $\left(\mathrm{HCO}_{3}\right)$ /carbonate $\left(\mathrm{CO}_{3}\right)$.

Using published information, it was shown that if aqueous solutions are present above the boiling point of pure water (due to hygroscopic salts), then the presence of oxygen partial pressure results in dissolved oxygen. The amount of dissolved oxygen affects the electrochemical potential, Eh, which in turn is a determinant of electrochemical corrosion processes.

Under conditions of very low partial pressure of $\mathrm{CO}_{2(g)}$, bicarbonate/carbonate waters can obtain very high $\mathrm{pH}$ values. A consequence of the high $\mathrm{pH}$ conditions is the very high solubility of silica. This was seen under laboratory conditions. Whether the conditions necessary for the formation of the high-pH solutions are operable under potential repository conditions needs further investigation.

Experimental studies show that the initial concentrating of the waters causes $\mathrm{Ca}$ and $\mathrm{Mg}$ precipitates to form. Effectively, the solutions are then chloride, fluoride, nitrate, sulfate, and bicarbonate/carbonate salts of sodium and potassium. Further concentrating these waters removes the fluoride ions from solution and decreases the sulfate concentration relative to the chloride and nitrate. The maximum boiling points of the concentrated solutions to date is $108^{\circ} \mathrm{C}$.

The characteristics of the aqueous solutions that are in contact with the EBS components, DSs, and WPOBs depend upon the temperature of the component, the relative humidity of the air at the component, and nature of the hygroscopic salts deposited on the component. The most prevalent hygroscopic salts expected to be deposited on the EBS components are nitrate salts of potassium and sodium. The solubilities of both salts are strongly temperature-dependent, which effects the hygroscopic properties of these salts. In particular, their decreasing solubility with temperature indicates an increasing deliquescence point with decreasing temperature. Experimental work is in progress to characterize the nature of the hygroscopic salts in this temperature/relative humidity region. 


\section{CHECK COPY}

\section{REFERENCES}

Cowan, J.C.; and Weintritt, D.J. 1976. Water-Formed Scale Deposits, Houston, TX: Gulf

Publishing Company. TIC:

Cramer, S.D.; 1980. "The Solubility of Oxygen in Brines from 0 to $300^{\circ} \mathrm{C}$," Ind. Engineering Chemical Process Des. Dev., 19, 300-305. Washington, D.C.: American Chemical Society. Vol. TIC: 244500 .

Fyfe, D. 1994,."The Atmosphere," Corrosion; Volume 1; Metal/Environment Reactions, L.L. Shreir, R.A. Jarman, G.T. Burstein, eds., Oxford, England: Butterworth-Heinemann Ltd., Section 2.2, pp. 2:31-2:42. TIC:

Frazier, J.C.W.; Taylor, R.K.; and Grollman, A. 1928. "Two-Phase Liquid-Vapor Isothermal Systems, Vapor-Pressure Lowering," International Critical Tables of Numerical Data, Physics, Chemistry and Technology, Washburn, E.W. ed., New York, NY: McGraw-Hill Book Company, Inc., p. 292, 297.

Greenspan, L.; 1977. "Humidity Fixed Points of Binary Saturated Aqueous Solutions" Journal of Research of the National Bureau of Standards - A. Physics and Chemistry, 81A, 89-96. TIC: 241138.

Harrar, J.E.; Carley, J.F.; Isherwood, W.F.; and Raber, E.; 1990. "Report of the Committee to Review the Use of H-13 Well Water in Nevada Nuclear Waste Storage Investigations" Report UCID-21867, Livermore, California: Lawrence Livermore National Laboratory. TIC: 209096.

Lee, S.; and Staehle, R.W. 1997. "Adsorption of Water on Copper, Nickel, and Iron" Corrosion, 53, 33-42. Houston, TX: National Association of Corrosion Engineers. TIC: 244501.

Perry, R.H.; and Chilton, C.H.; eds., 1973. Chemical Engineers' Handbook $5^{\text {th }}$ Edition, New York, NY: McGraw-Hill Book Company. TIC: 242591.

Stumm, W.; and Morgan, J.J. 1981. Aquatic Chemistry; An Introduction Emphasizing Chemical Equilibria in Natural Waters, $2^{\text {nd }}$ Edition. New York, NY: John Wiley \& Sons, Inc. TIC: 208448.

Weast, R.C.; and Astle, M.J. 1981. CRC Handbook of Chemistry and Physics $62^{\text {nd }}$ Edition 19811982, Boca Raton, FL: CRC Press, Inc., D-168-D-169. TIC: 240722.

CRWMS M\&O 1999. Uncanistered Spent Nuclear Fuel Disposal Container System Description Document. BBA000000-01717-1705-00004 Rev 01. Las Vegas, NV: CRWMS M\&O. ACC: MOL.

Department of Energy (DOE) 1998a. Quality Assurance Requirements and Description. DOE/RW-0333P, Rev 8. Washington, DC: U.S. Government Printing Office. ACC: MOL.19980601.0022. 
$$
\text { ' }
$$ 


\section{ATTACHMENTS}

Attachments to this document are listed in Table 9-1.

Table 9-1. List of Attachments

\begin{tabular}{|c|c|c|}
\hline $\begin{array}{c}\text { Attachment } \\
\text { Number }\end{array}$ & Description & Size \\
\hline$I$ & & \\
\hline$I I$ & & \\
\hline$I I I$ & & \\
\hline$I V$ & & \\
\hline$V$ & & \\
\hline$V I$ & & \\
\hline$V I I$ & & \\
\hline$V I I I$ & & \\
\hline$I X$ & & \\
\hline$X$ & & \\
\hline
\end{tabular}

The following supporting documents are in electronic form on a CD-ROM. Each file is identified by it's name, size (in bytes), and the date and time of last access.

DOS Filename byte size date time WIN95 filename 
